\title{
Passivity Preserving Model Order Reduction Using the Reduce Norm Method
}

\author{
Namra Akram ${ }^{1}$, Mehboob Alam ${ }^{2}{ }^{(0)}$, Rashida Hussain ${ }^{1}{ }^{(0}$, Asghar Ali $^{1}$, Shah Muhammad ${ }^{3}$, \\ Rahila Malik ${ }^{1}$ and Anwar Ul Haq ${ }^{4, *(1)}$ \\ 1 Department of Mathematics, Mirpur University of Science and Technology (MUST), Mirpur 10250, Pakistan; \\ namra.math@gmail.com (N.A.); drrashida@must.edu.pk (R.H.); drali@must.edu.pk (A.A.); \\ rahila.maths@gmail.com (R.M.) \\ 2 Department of Electrical Engineering, Mirpur University of Science and Technology(MUST), Mirpur 10250, \\ Pakistan; m.alam@ieee.org \\ 3 Department of Mathematics, King Saud University, Riyadh 11451, Saudi Arabia; skabeer@ksu.edu.sa \\ 4 Department of Informatics (I-13), Bolzmanstr. 3, Technical University Munich, 85748 Garching, Germany \\ * Correspondence: anwar.haq@tum.de
}

Received: 17 April 2020; Accepted: 4 June 2020; Published: 9 June 2020

\begin{abstract}
Modeling and design of on-chip interconnect, the interconnection between the components is becoming the fundamental roadblock in achieving high-speed integrated circuits. The scaling of interconnect in nanometer regime had shifted the paradime from device-dominated to interconnect-dominated design methodology. Driven by the expanding complexity of on-chip interconnects, a passivity preserving model order reduction (MOR) is essential for designing and estimating the performance for reliable operation of the integrated circuit. In this work, we developed a new frequency selective reduce norm spectral zero (RNSZ) projection method, which dynamically selects interpolation points using spectral zeros of the system. The proposed reduce-norm scheme can guarantee stability and passivity, while creating the reduced models, which are fairly accurate across selected narrow range of frequencies. The reduced order results indicate preservation of passivity and greater accuracy than the other model order reduction methods.
\end{abstract}

Keywords: model order reduction; passivity; spectral-zeros; krylov subspace; integrated circuit; reduce norm; VLSI; interconnect

\section{Introduction}

In large-scale complex systems, it is often desirable to use a set of mathematical equations to represent the behavior of the systems [1,2]. Over the last few decades, the continuous scaling of on-chip devices and interconnect resulted in exponential increase in the complexity of modeling and simulation [3,4]. The automated design of on-chip interconnect is important in meeting the increasing demand of modern high-speed electronics [5,6]. Over the past decade, the rapid advancement in both device and interconnect design automation have presented a great challenge. The complex and large design space of on-chip interconnect necessitates efficient modeling techniques [7-9]. Model order reduction (MOR) is essential to overcome the exceptional computational resource requirements of full simulations of the integrated circuit (IC) [10]. The motivation is the desire to develop simplified models, which reduce the simulation time and also capture the main properties of the complex original system. For large scale on-chip interconnect problems, our work aims at developing efficient reduced order model (ROM), which will accelerate simulation and provide rapid design optimization of the system.

MOR methods, which are widely used and successful for large-scale linear systems, fall into two main types, i.e., projection and non-projection based methods [11-13]. Krylov-subspace and singular 
value decomposition (SVD) based methods are the well-known projection methods. In non-projection based methods, Hankle-norm approximation is a popular example of MOR method [14]. In developing $\mathrm{ROM}$, there are multiple requirements to be satisfied, while approximating the original system. The reduced number of state variables and accurate representation of the original system by correctly following the terminal behavior are two such important factors $[15,16]$. In this work, we will only consider stable and passive interconnect systems, as a result making it desirable that reduced models preserve the important properties of stability and passivity. It is observed that an original stable but not passive system, once coupled with other passive components, can make the system unstable [17]. Passivity preserving model order reduction for the on-chip interconnect systems mainly uses two main methods. The first uses SVD-based balance approximation and the other estimates ROMs via Krylov subspace methods [18-20]. It is observed here that for large systems, the approximation using the Krylov subspace projection gives a good estimation of ROM compared to SVD-based methods [21].

Passivity preservation MOR via Krylov subspace method with congruence transformation includes passive reduced-order interconnect macromodeling algorithm (PRIMA) [19] and its variant SPRIM [22]. Generally, projection methods use reachability subspaces, constructed using sequence of vectors. These techniques are basically moment matching of the systems and gives an, iterative approximation of the eigenvalues. There are various algorithms based on Krylov subspace projection method [23-25]. Computationally, these methods are less expensive and their, iterative nature makes them attractive as compared to balanced approximations [26,27]. However, these Krylov based approximation methods match one moment per iteration and sometime may result in low-accuracy [28]. In addition, unlike balanced approximation methods, the stability is not always assured and the system approximation is also without an upper error bound. Passivity preserving methods based on balanced approximations such as PMTBR [29,30], PRTBR [31] and FABT [32,33] are SVD based techniques. These techniques require dense computations of the order of $n^{2}$ and $n^{3}$ and may not be suitable for ROM of large-scale systems [34]. Another approach of passivity preservation model order reduction uses spectral zeros (SZ) as positive real interpolation points to produce ROM with roots as its SZs [35,36]. An efficient scheme to implement this techniques was later proposed, which uses certain block matrix with SZs as its spectrum [37]. The selection criteria of SZs for optimal matching in these schemes is still not obvious. A selection criteria based on dominant spectral zeros was later suggested, which uses the magnitude of the associated residues of the system dominant poles [38]. However, the selection of SZs using dominant pole is computationally expensive.

In this work, we focus on solving complex problems in the design of modern processors, which operate on dual frequencies to account for its low power as well as turbo mode operations. Note that the proposed scheme is for high-speed ICs, where chip operates at GHz frequency range with no more than 1-3 GHz difference between their dual mode frequencies. In these high-speed ICs, the scaling of features significantly influence the performance [39], especially with increasing processor frequencies. This results in design automation focused research efforts in the MOR $[19,40,41]$. In this paper, we propose a frequency selective reduce norm spectral zero (RNSZ) projection method, which uses a simple reduce-norm criteria to select interpolation points using SZs of the system. In the proposed method, the frequency of interest is divided into regular intervals and interpolation points are the selected SZ, which provides the best estimation in the minimum-norm sense. The result is the preservation of passivity and stability properties in the ROM. It is important to note that selected interpolation points are part of the stable SZ and therefore guarantee passivity of the reduced system. The simulation results of ROM using proposed method show more accurate approximations with less absolute error for the selected narrow band of frequencies compared to other model order reduction methods.

In next section, we discuss MOR and important passivity preserving reduction techniques. The proposed reduced-norm frequency selective technique is explained in Section 3. Section 4 discusses the simulation and analysis of the results and the paper is finally concluded in Section 5 . 


\section{Model Order Reduction of On-Chip Interconnects}

Recent advances in the IC fabrication ultimately lead to the transformation of the computer-aided design (CAD) industry [10]. The scaling of technology over a period of time has shifted the design paradigm to interconnect dominated methodology. In the CAD tools, MOR reduces the design complexity of the numerical simulation [42]. In a RLC (resistor, inductor, capacitor) network model of an on-chip interconnect, preserving of the passivity inherent in the system is essential [10]. It is important to note that all passive systems are stable but on the contrary all stable systems are not guaranteed to be passive [17]. This section discusses the preliminaries of model order reduction using projection with special emphasis to passivity preserving techniques for on-chip interconnects.

\subsection{State Description and Mor Using Projection}

In the state space model of the on-chip interconnect, the original system of order $n$ in descriptor-form is given as

$$
\begin{aligned}
\mathbf{E} \dot{\mathbf{x}} & =\mathbf{A x}(\mathbf{t})+\mathbf{B u}(\mathbf{t}) \\
\mathbf{y}(\mathbf{t}) & =\mathbf{C x}(\mathbf{t})+\mathbf{D u}(\mathbf{t})
\end{aligned}
$$

where $\mathbf{x}(\mathrm{t}) \in \mathbb{R}^{n}$ represents the internal states of the system, $\mathbf{u}(\mathrm{t}) \in \mathbb{R}^{m}$ and $\mathbf{y}(\mathrm{t}) \in \mathbb{R}^{p}$ are the system input and output respectively. Correspondingly, $\mathbf{E}, \mathbf{A} \in \mathbb{R}^{n \times n},(\mathbf{A}, \mathbf{E})$ are Generalized EigenValues (GEV), $\mathbf{B} \in \mathbb{R}^{n \times m}, \mathbf{C} \in \mathbb{R}^{p \times n}, \mathbf{D} \in \mathbb{R}^{p \times m}$ are the matrices defining linear maps between inputs, output and internal states. It is important to note that the extracted model in the state space form shown in (1) accounts for all the resistance, inductance and capacitance effects of the on-chip interconnect. The block matrices of resistance (R), inductance (L) and capacitance (C) will appear in the state space as

$$
\begin{gathered}
\underbrace{\left[\begin{array}{l|l}
C & 0 \\
\hline 0 & L
\end{array}\right]}_{E} \dot{x}(t)=\underbrace{\left[\begin{array}{l|l}
R & \\
\hline & 0
\end{array}\right]}_{A} x(t)+\underbrace{\left.[]_{B}\right]}_{B} u(t), \\
y(t)=\underbrace{\left.[]_{D}\right]}_{B^{T}=C} x(t)+\underbrace{[]}_{D} u(t),
\end{gathered}
$$

The associated $\operatorname{ROM} \hat{\Sigma}(\hat{\mathbf{E}}, \hat{\mathbf{A}}, \hat{\mathbf{B}}, \hat{\mathbf{C}}, \mathbf{D})$ for (1) of order $k$ is given as

$$
\begin{aligned}
\hat{\mathbf{E}} \hat{\mathbf{x}} & =\hat{\mathbf{A}} \hat{\mathbf{x}}(\mathbf{t})+\hat{\mathbf{B}} \mathbf{u}(\mathbf{t}), \\
\hat{\mathbf{y}}(\mathbf{t}) & =\hat{\mathbf{C}} \hat{\mathbf{x}}(\mathbf{t})+\mathbf{D u}(\mathbf{t}),
\end{aligned}
$$

where $\hat{\mathbf{x}} \in \mathbb{R}^{k}, \hat{\mathbf{E}} \in \mathbb{R}^{k \times k}, \hat{\mathbf{A}} \in \mathbb{R}^{k \times k}, \hat{\mathbf{B}} \in \mathbb{R}^{k \times m}, \hat{\mathbf{C}} \in \mathbb{R}^{p \times k}, \mathbf{D} \in \mathbb{R}^{p \times m}$ with $\mathrm{k}<<\mathrm{n}$. As noted earlier, the design automation tools require that the reduced models should preserve important properties of stability and passivity of the original system. The theory of stability plays an important role in the MOR. In dynamical system, the concept is explained in terms of internal and external stability of the system. Similarly, for the passivity, a passive ROM does not generate any energy. Passivity in ROM is sometime ensured by positive real transfer function [15].

In projection based MOR methods consisting of the projection matrices $\mathbf{V}$ and $\mathbf{W}$, the ROM is obtained such that $\mathbf{V}, \mathbf{W} \in \mathbb{R}^{n \times k}$ and $\mathbf{W}^{\mathbf{T}} \mathbf{V}=\mathbf{I}_{\mathbf{k}}$. A careful selection of $\mathbf{V}$ and $\mathbf{W}$ by properly deciding projection subspace ensures that ROM not only preserves the stability and passivity, but also retains the dynamical characteristics of the original response. The reduced system matrices using these projectors are represented as:

$$
\hat{\mathbf{E}}=\mathbf{W}^{\mathrm{T}} \mathbf{E V}, \quad \hat{\mathbf{A}}=\mathbf{W}^{\mathrm{T}} \mathbf{A V}, \quad \hat{\mathbf{B}}=\mathbf{W}^{\mathrm{T}} \mathbf{B}, \quad \hat{\mathbf{C}}=\mathbf{C V} .
$$


The accuracy of ROM is completely determined by the choices of the projection matrices $\mathbf{V}$ and $\mathbf{W}$. These projection martices control the way system properties are preserved by the reduced system and also, identifies the accuracy of the ROM. It is important to note that in the applications of circuit simulation, both invertible and non-invertible matrix $\mathbf{E}$ arises in the system simulation. A singular matrix $\mathbf{E}$ give rise to new set of challenges as some of the system poles and SZs lies at infinity. In MOR, invertible and non-invertible $\mathbf{E}$ are handled differently [43,44]. However in this work, $\mathbf{E}$ is an invertible non-singular matrice, as it appears in the state representation of on-chip interconnects.

\subsection{Passivity Preserving Mor}

Passivity of the LTI system is preserved if the transfer function $\mathrm{G}(\mathrm{s})$ is positive real [15]. This means $\mathrm{G}(\mathrm{s})$ satisfies the following conditions

- $\quad \mathrm{G}(\mathrm{s})$ is analytic for $\operatorname{Real}(\mathrm{s})>0$;

- $\mathbf{G}(\bar{s})=\overline{\mathbf{G}(s)}$ for all $\mathrm{s} \in \mathbb{C}$;

- $\mathrm{G}(\mathrm{s})+\mathrm{G}(-\mathrm{s})^{T} \geq 0$ for $\operatorname{Real}(\mathrm{s})>0$.

Therefore, for real systems, we always have $\mathbf{G}(\bar{s})=\overline{\mathbf{G}(s)}$ and the last condition shows the presence of stable matrix function $\mathbf{V}(\mathbf{s})$ (inverse exists) such that $\mathbf{G}(\mathbf{s})+\mathbf{G}^{\mathbf{T}}(-\mathbf{s})=\mathbf{V}(\mathbf{s}) \mathbf{V}^{\mathbf{T}}(-\mathbf{s})$ is the spectral factorization of $\mathbf{G}$, where $\mathbf{V}$ is the spectral factor of $\mathbf{G}$. The $\mathbf{S Z s}$ of $\mathbf{G}$ are obtained from the zeros of $\mathbf{V}$, i.e., $\Lambda_{i}$, for $i=1, \cdots, n$, such that $\operatorname{det} \mathbf{V}\left(\Lambda_{i}\right)=0$.

This section defined the passivity preserving in the MOR and existence of stable rational function, which helps in obtaining SZs of the system. In the next section, we have proposed a scheme, which selects a subset of these SZs as interpolation points to give best estimation of system approximation in the minimum-norm sense. The result is a ROM, which preserves the passivity and stability of the system.

\section{Passivity Preserving MOR Using RNSZ}

This section defines the procedure of MOR using reduce-norm spectral zero (RNSZ) method. It is a frequency selective reduction method, which uses subset of $\mathrm{SZ}$ as interpolation point using simple reduce-norm criteria to construct projection matrices. The result is an optimally reduced system, which also preserves the essential properties of the original system.

\subsection{Passivity Preserving Projection}

The RNSZ method establishes relationship of the selected SZ with the system spectral zeros. However, it is desirable first to discuss some basic parameters of the system and its properties, which are used in the derivation. Given the descriptor system (1), the Laplace transform of the transfer function $\sum$ is given as

$$
\mathbf{G}(\mathbf{s})=\mathbf{C}(\mathbf{s E}-\mathbf{A})^{-\mathbf{1}} \mathbf{B}+\mathbf{D} .
$$

Note that the poles of the system $\sum$ are the GEVs of the pair $(\mathbf{A}, \mathbf{E})$. Let the matrix $(\mathbf{A}-\mathbf{\Lambda E})$ be non-singular throughout for some $\Lambda \in \mathbb{C}$ [45], which is represented as a matrix pencil (A, E) in mathematical terms. Then the system $\sum$ is said to be stable if and only if all finite poles are lying in the left half of the $s$ plane, i.e., $\operatorname{Re}(\Lambda)<0$ with $|\Lambda| \neq \infty$. In this connection, it turns out that the SZs of $\Sigma$ are all $\mathbf{s} \in \mathbb{C}$, such that $\operatorname{det}\left[\mathbf{G}(\mathbf{s})+\mathbf{G}^{\mathbf{T}}(-\mathbf{s})\right]=0$, where

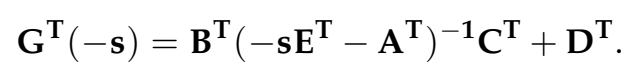

This definition shows that each SZ has the reflection in the $s$ plane. Note that the Real SZs $s_{i}$ are in pairs $\left(\mathrm{s}_{i},-\mathrm{s}_{i}\right)$, whereas complex exist in quadruples $\left(\mathrm{s}_{i}, \mathrm{~s}_{i}^{T},-\mathrm{s}_{i},-\mathrm{s}_{i}^{T}\right)$. Alternately, these definitions also explain the positive realness of the transfer function and if SZs are used to drive projection matrices, the system preserves the passivity of the ROM. 
Essentially, this is the motivation of our proposed frequency selective passivity preserving RNSZ method. The method divides the frequency of interest in to regular intervals and selects the SZs, which minimizes the norm of the distance. The selected SZs become the interpolation point for constructing projection matrices ( $\mathbf{V}$ and $\mathbf{W}$ ), which simultaneously assure stability and passivity of the system, while creating the frequency-selective ROM. In the proposed RNSZ method, we first constructed the following matrix $\bar{G}$ and $\bar{E}$ from the state representation of system given by (1).

$$
\bar{G}:=\left[\begin{array}{ccc}
\mathbf{A} & & \mathbf{B} \\
& -\mathbf{A}^{\mathbf{T}} & -\mathbf{C}^{\mathbf{T}} \\
\mathbf{C} & \mathbf{B}^{\mathrm{T}} & \mathbf{D}+\mathbf{D}^{\mathrm{T}}
\end{array}\right],
$$

and

$$
\overline{\mathrm{E}}:=\left[\begin{array}{lll}
\mathbf{I} & & \\
& \mathbf{I} & \\
& & \mathbf{0}
\end{array}\right] .
$$

These matrices are used for extracting the finite eigenvalues of a GEV problem. In this formulation, the finite SZs (subset of eigenvalues) of $\bar{G}$ are all complex numbers $\Lambda$, such that Rank $(\overline{\mathrm{G}}-\Lambda \overline{\mathrm{E}})<2 \mathrm{n}+$ p, i.e., it is equivalent to solving the finite GEVs $\Lambda(\overline{\mathrm{G}}, \overline{\mathrm{E}})$. The pair, therefore, provides the desired passivity preserving projection vectors $\mathbf{V}$ and $\mathbf{W}$ to maintain the passivity of the reduced model. Note that solving the generalized eigenvalue problem generates eigenvalues and eigenvectors necessary for finding the SZs.

\subsection{Spectral Zero Selection and Iterative Algorithm for RNSZ}

The preceding section explained the passivity preserving projection followed by the important task of constructing matrices $\mathbf{V}$ and $\mathbf{W}$. The projection matrices are determine by the selected SZs, which are the subset of the $n$ stable SZs of the original system. It is observed here that the proposed method for the selection of SZs is associated to the minimum norm value of the SZs. The selected SZs characterize the system's output and their use as interpolation points determine the accuracy of the ROM system. The pseudocode giving details of the proposed RNSZ method in selecting the spectral zeros is given in Algorithm 1. Using examples from [37], the partial fraction expansion of $\mathbf{G}(\mathbf{s})$ can be written as rational function $\mathbf{G}(\mathbf{s})+\mathbf{G}^{\mathbf{T}}(-\mathbf{s})$ and is used to compute the stable spectral zeros. In the first step, $[Q, R]$ factorization are taken into account for calculating the eigenvalues and eigenvector matrices of the system. Note that SZs of $\sum$ are the GEVs of the following structured eigenvalue problem.

$$
(\bar{G}-R \bar{E}) Q=0,
$$

where $\bar{G}$ and $\bar{E}$ are given by (6) and (7) respectively. In Equation (8), $Q$ and $R$ represent the matrices of the eigenvectors and eigenvalues, whereas SZs are the diagonal elements of $R$. Note that the formulation of $(\bar{G}, \bar{E})$ pair, ensures that the eigenvalues, which are the SZs of the system, lay on the left half plane. In our case $\mathbf{D}+\mathbf{D}^{\mathbf{T}}$ is singular, which is equivalent to solving a generalized eigenvalue problem for the computation of SZs. Therefore, the GEVs of $\Lambda(\overline{\mathrm{G}}, \overline{\mathrm{E}})$ are the SZs of the original system.

It is important to note that the RNSZ method is a frequency selective approximation of large-scale dynamical system. Considering SZs of $\sum$ be the poles of $\mathbf{G}(\mathbf{s})$, we suggest a criterion to choose SZs on the basis of dominance of each SZs in the frequency of interest. Therefore, the proposed method introduces a reduce-norm criteria for the selection of SZs of the system transfer function $\mathbf{G}(\mathbf{s})$. Motivation is to solve complex problem of model order reduction of on-chip interconnects of a modern processor. We take the example of a Intel Core i7-2920XM processor (Intel, Santa Clara, CA, USA), which operates on dual frequencies $\left(f_{1}\right.$ and $\left.f_{2}\right)$ to account for its low power as well as turbo mode 
operations [46]. The frequency selection is performed by uniformly distributing the frequency of interest. The $k$ th frequency interval between the low and high frequency can then be defined as

$$
f_{k}=f_{1}+k\left(\frac{f_{2}-f_{1}}{N}\right)
$$

where $N$ is the order of the ROM, with $f_{1}$ and $f_{2}$ defining selected frequency range with $f_{2}>f_{1}$. Therefore, in the total $n$ stable SZs, the $k$ th selected SZ is chosen based on following reduce norm criteria

$$
\mathrm{SZ}_{k}=\left|\left\|f_{k}\right\|-\|\operatorname{imag}(S Z)\|\right|_{\min }
$$

Note that the selected interpolation points are SZs, which reduce the minimum norm distance between the selected frequency interval and the distributed SZs in the complex spectral domain. In this reduction, the $N$ selected SZs form the basis of developing ROM, with corresponding eigenvectors of the selected $S Z$ given by $\left[Q_{x}, S, Q_{y}\right]=\operatorname{svd}\left(X^{T} Y\right)$. The eigenvectors of the selected $S Z s$ are then used in constructing the projection vectors $\mathrm{V}$ and $\mathrm{W}$ of the system as

$$
\mathrm{V}=\mathrm{XQ}_{x} \mathrm{~S}^{-1} \text {, and } \mathrm{W}=\mathrm{YQ}_{y} \mathrm{~S}^{-1} \text {. }
$$

Note that these projection matrices not only dynamically generate ROM but also satisfy the orthogonality condition. This is a generalization of the approximation by projection using our proposed RNSZ method. We can now directly state the fundamental results of RNSZ by the following theorem.

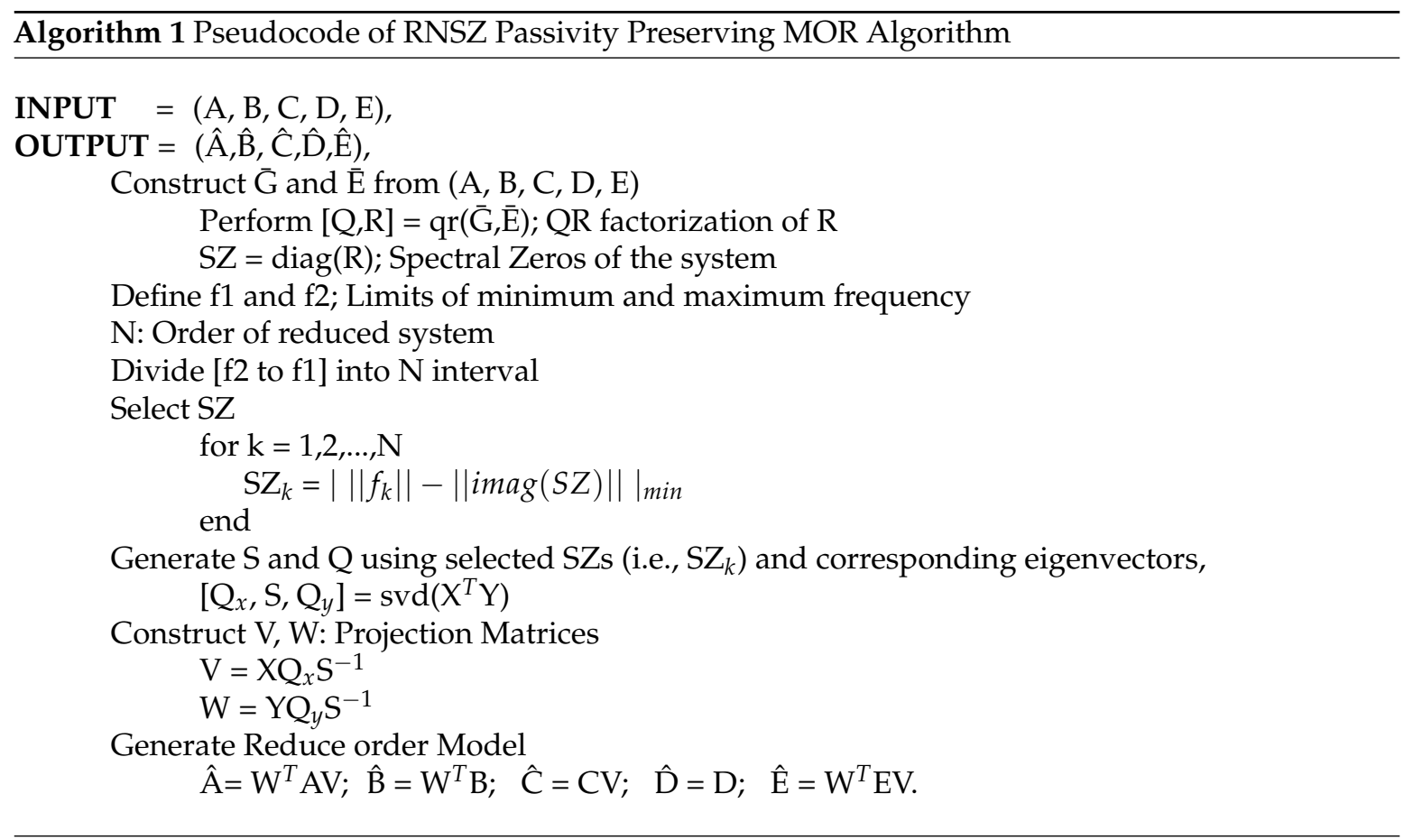

Theorem 1. Given a descriptor system defined by (1), the transfer function of $\sum(A, B, C, D, E)$ is $G(s)=C(s E-A)^{-1} B+D$ is positive real. The system $S Z$ s are the required interpolation points using criteria $S Z_{k}=\left|\left\|f_{k}\right\|-\|\operatorname{imag}(S Z)\|\right|_{\text {min }}$ for $k=1,2,3, \ldots, N$ in the frequency interval $f_{k}=f_{1}+k\left(\frac{f_{2}-f_{1}}{N}\right)$, where $N$ is the order of ROM. The reduced norm system $\hat{\sum}(A, B, C, D, E)$ is defined by $\hat{A}=W^{T} A V, \hat{B}=W^{T} B, \hat{C}=C V$, $\hat{D}=D, \hat{E}=W^{T} E V$ and $\hat{G}(s)=\hat{C}(s \hat{E}-\hat{A})^{-1} \hat{B}+\hat{D}$ are both passive and stable. 
Next, we explain the implementation of RNSZ method by using a basic example of RLC ladder network.

Example 3.1: Consider Figure 1 RLC Network [37]. Let us define $x_{1}, x_{3}$ and $x_{5}$ be the voltage across $C_{1}, C_{2}$ and $C_{3}$ respectively, whereas the current across $L_{1}$ and $L_{2}$ are represented by $x_{2}$ and $x_{3}$ respectively. The input voltage $u$ and output current $y$ are also defined in (1). The capacitors and inductors have unity value, while $R_{1}=\frac{1}{2}$ and $R_{2}=\frac{1}{5}$. The rest of the example using RNSZ method is solved as follows.

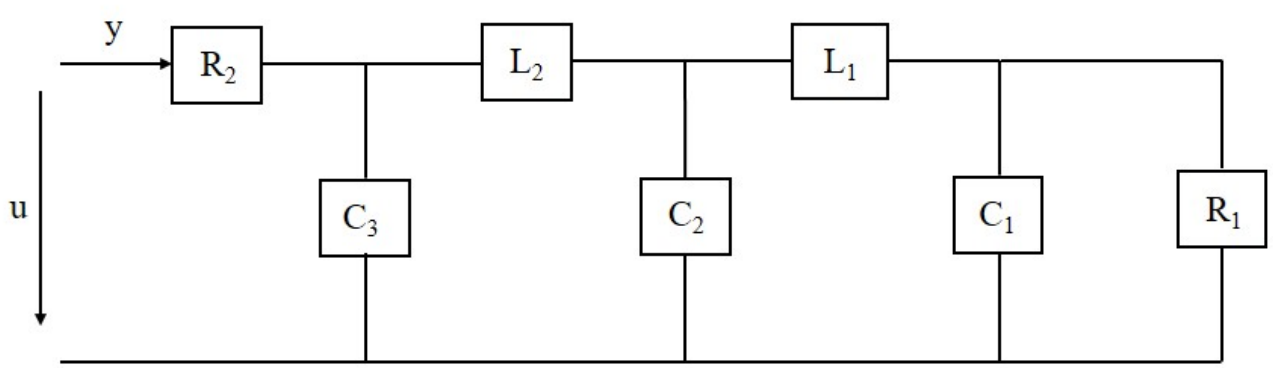

Figure 1. RLC ladder circuit consisting of resistors, inductors and capacitors forming a 5th order system.

The transfer function $G(s)$ of the RLC circuit given by Figure 1 can be written as

$$
G(s)=\frac{s^{5}+3 s^{4}+6 s^{3}+9 s^{2}+7 s+3}{s^{5}+7 s^{4}+14 s^{3}+21 s^{2}+23 s+7} .
$$

The partial fraction expansion in the form of rational function is given as

$$
G(s)+G^{T}(-s)=\frac{2\left(s^{10}-s^{8}-12 s^{6}+5 s^{4}+35 s^{2}-21\right)}{\left(s^{5}+7 s^{4}+14 s^{3}+21 s^{2}+23 s+7\right)\left(s^{5}-7 s^{4}+14 s^{3}-21 s^{2}+23 s-7\right)} .
$$

Minimal realization of the state matrices of $G(s)(12)$ is given as

$$
\begin{gathered}
A=\left[\begin{array}{ccccc}
-2 & 1 & 0 & 0 & 0 \\
-1 & 0 & 1 & 0 & 0 \\
0 & -1 & 0 & 1 & 0 \\
0 & 0 & -1 & 0 & 1 \\
0 & 0 & -0 & -1 & -5
\end{array}\right], B=\left[\begin{array}{l}
0 \\
0 \\
0 \\
0 \\
2
\end{array}\right], \\
C=\left[\begin{array}{lllll}
0 & 0 & 0 & 0 & -2
\end{array}\right], E=\left[\begin{array}{ccccc}
1 & 0 & 0 & 0 & 0 \\
0 & 1 & 0 & 0 & 0 \\
0 & 0 & 1 & 0 & 0 \\
0 & 0 & 0 & 1 & 0 \\
0 & 0 & 0 & 0 & 1
\end{array}\right], D=1 .
\end{gathered}
$$

Using reduce norm selection criteria $\mathrm{SZ}_{k}=|| \mid f_{n}(k)\|-\|$ imag $\left.(S Z)||\right|_{\text {min }}$, we have selected three spectral zeros, i.e., $[0.1833+1.5430 \mathrm{i}, 0.1833-1.5430 \mathrm{i},-0.1833+1.5430 \mathrm{i}]$ to construct projection matrices $\mathbf{V}$ and $\mathbf{W}$ using (11). Using these projectors, we can generate following ROM.

$$
\begin{gathered}
A_{n}=\left[\begin{array}{ccc}
2.8629 & -3.9654 & 4.0350 \\
-1.0999 & 2.2405 & -3.5414 \\
0.2158 & -0.2190 & 1.5778
\end{array}\right], B_{n}=\left[\begin{array}{c}
0.9925 \\
-0.6084 \\
0.1270
\end{array}\right], \\
C_{n}=\left[\begin{array}{lll}
2.3915 & -2.3852 & 2.3852
\end{array}\right],
\end{gathered}
$$




$$
E_{n}=\left[\begin{array}{lll}
1 & 0 & 0 \\
0 & 1 & 0 \\
0 & 0 & 1
\end{array}\right], D_{n}=1
$$

\section{Simulation and Analysis}

This section discuses the accuracy and efficiency of our proposed RNSZ method. We take examples of on-chip interconnect structures including interconnect bus and a RLC network. The models are created using the field solvers $[47,48]$ for capacitance and inductance extraction. A combination of modified nodal analysis is then used to create state space representation as described in (1). For the purpose of checking the accuracy of our proposed RNSZ method, we generated examples of an order 79 and 1079 RLC network and interconnect bus respectively. In addition, we also take a look at series of examples of on-chip spiral inductor with transfer function ranging from few $\mathrm{kHz}$ to $\mathrm{GHz}$. The examples covers the frequency ranges of $3 \mathrm{kHz}$ to $3 \mathrm{GHz}, 1 \mathrm{MHz}$ to $3 \mathrm{GHz}, 10 \mathrm{MHz}$ to $3 \mathrm{GHz}$ and $100 \mathrm{MHz}$ to $3 \mathrm{GHz}$ to discuss some limitations of the proposed RNSZ method. The results discussed at the end of this section highlight that RNSZ is suitable for application specific solution and its use may be restricted only for narrow-band high-speed ICs applications.

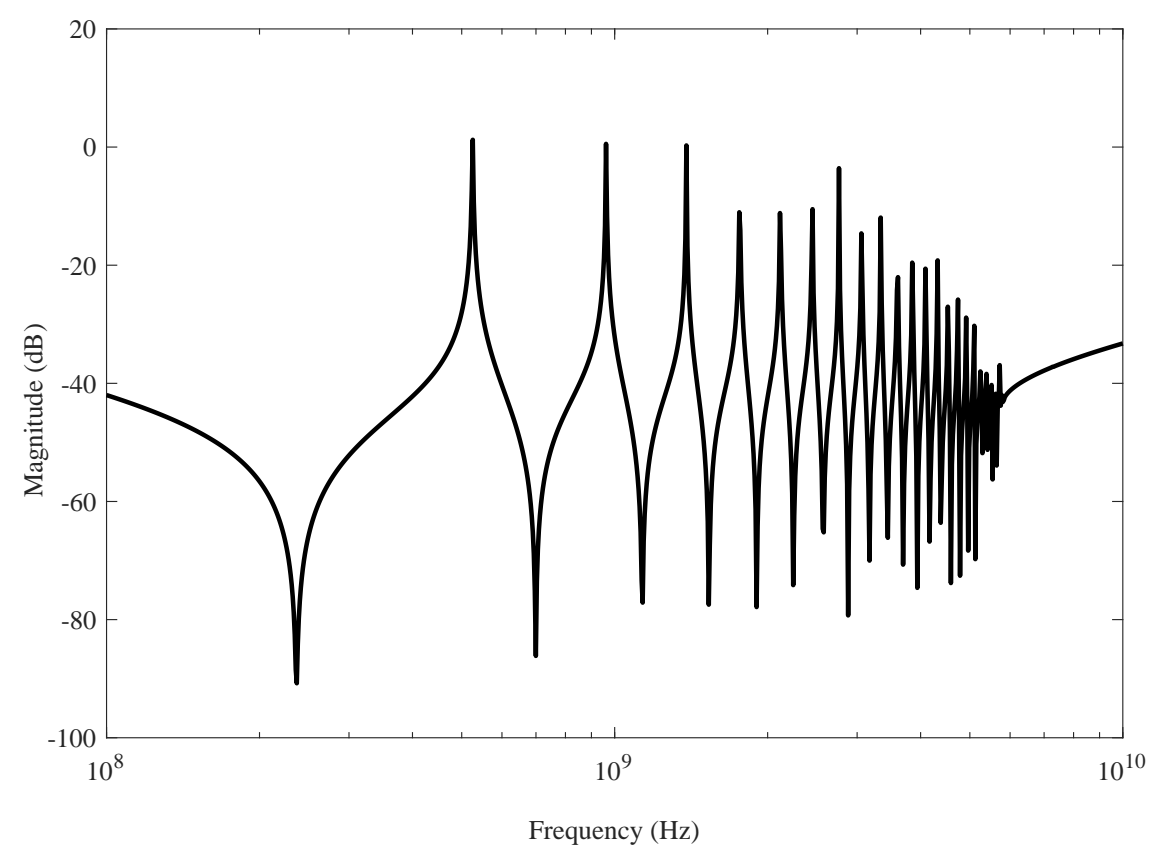

Figure 2. Original system frequency response of a on-chip 79 order RLC network.

Firstly, we took the example of a low order 79 RLC network and compared the reduce order modeling result of PRIMA [19] and interpolation via SZ [37] with RNSZ method. The original system of RLC network is plotted in Figure 2. The comparison results in Figures 3 and 4 (projection showing selected frequency of interest from $2.5 \mathrm{GHz}$ to $3.5 \mathrm{GHz}$ ) shows a close match of RNSZ, with an overlapping peaks and response compared to the original system. However, a significant deviation is observed in case of SZ method using interpolation at selected spectral zeros and PRIMA. A comparison of the absolute error in $\mathrm{dB}$ is plotted in Figure 5. The error plot shows that our proposed method of RNSZ method has considerably reduce error relative to the PRIMA and interpolation via SZ. 


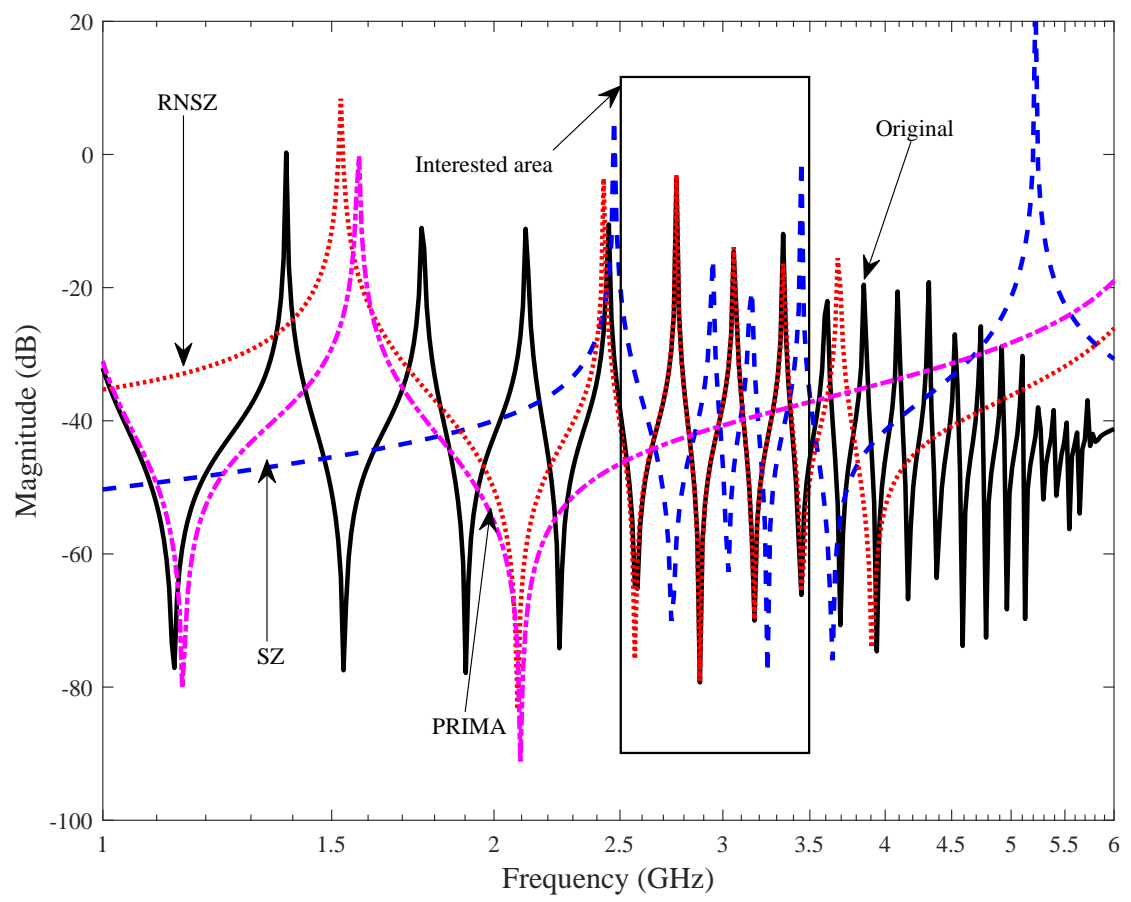

Figure 3. Frequency response of a on-chip RLC network with original system order of $n=79$. The reduce models of using reduce norm spectral zero (RNSZ), PRIMA and spectral zeros (SZ) methods are of order $\mathrm{N}=10$. The reduce model generated by RNSZ closely matches the fine details of the original system compared to other techniques.

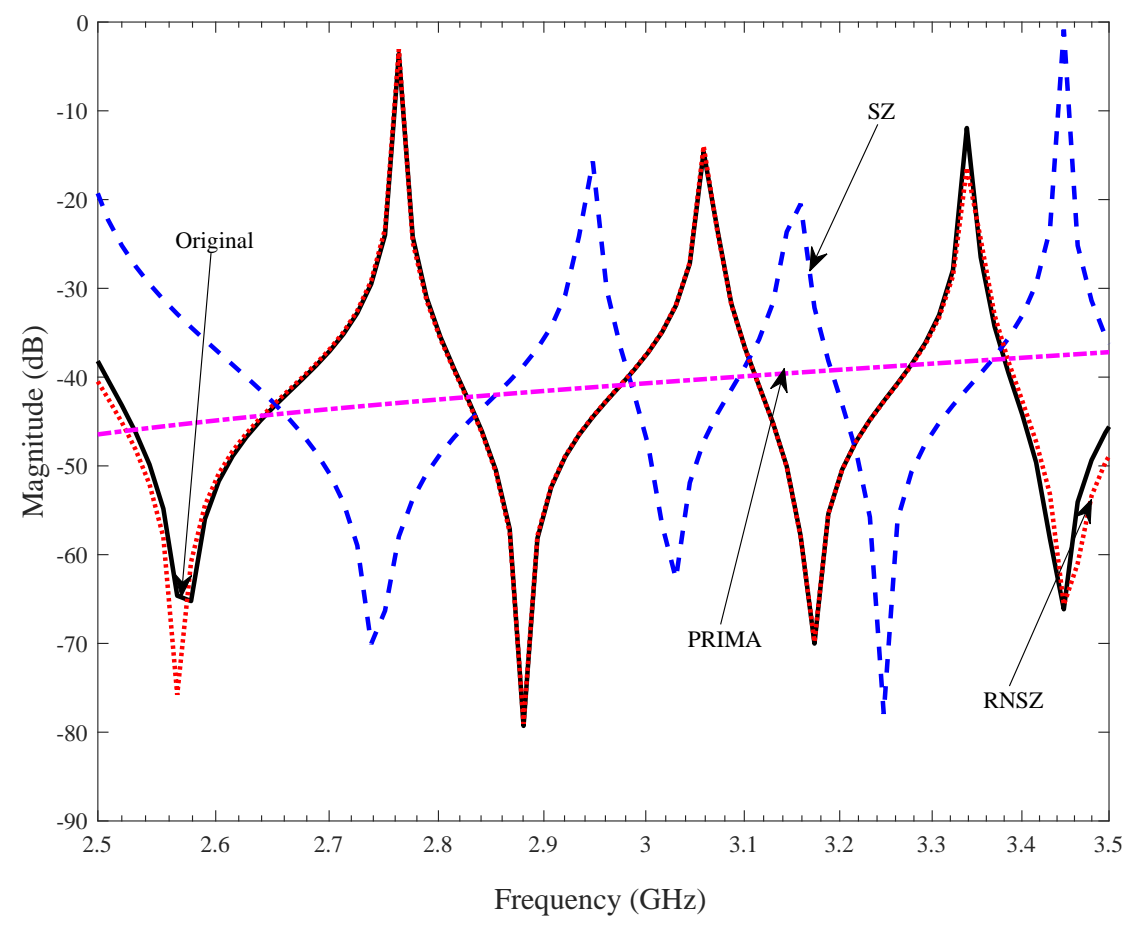

Figure 4. Magnitude response of the frequency of interest, i.e., operating frequency range of i7-2920XM from $2.5 \mathrm{GHz}$ to $3.5 \mathrm{GHz}$. The proposed RNSZ method closely matches with the original system. 
In order to compare complexity for large span of frequency, we also plotted the same RLC network example by increasing the span from $2 \mathrm{GHz}$ to $3.5 \mathrm{GHz}$ in Figure 6. In this case, note that the number of states required by RNSZ method doubled from $N=10$ to 20 . It is observed that the complexity of the amplitude response and the desired minimum error will determine the order of ROM, which considerably varies from example to example. The presence of resistor, inductor and capacitor in on-chip interconnect makes the response oscillatory and unique to each examples. The number of oscillation peaks and bottoms in a particular span of frequency is directly proportional to the complexity of the ROM of system. A higher order example of spiral inductor covering a large span of frequency, i.e., from $1.5 \mathrm{GHz}$ to $3.5 \mathrm{GHz}$ is discussed at the end of this section.

The second example is a state space model of an interconnect bus. The initial system response of the bus is plotted in Figure 7. The example is taken, keeping in mind the state of art the processor. The extraction tools creates this example keeping in mind Intel Core i7-2920XM modern processor [46]. Note that i7-2920XM works on two frequencies, i.e., $2.5 \mathrm{GHz}$ and $3.5 \mathrm{GHz}$, which actually defines the frequency of interest in this simulation. Figure 8 displays the magnitude response of original $n=$ 1076 order interconnect bus, with reduced order $N=100$ response using PRIMA [19], SZ [37] and proposed RNSZ method.

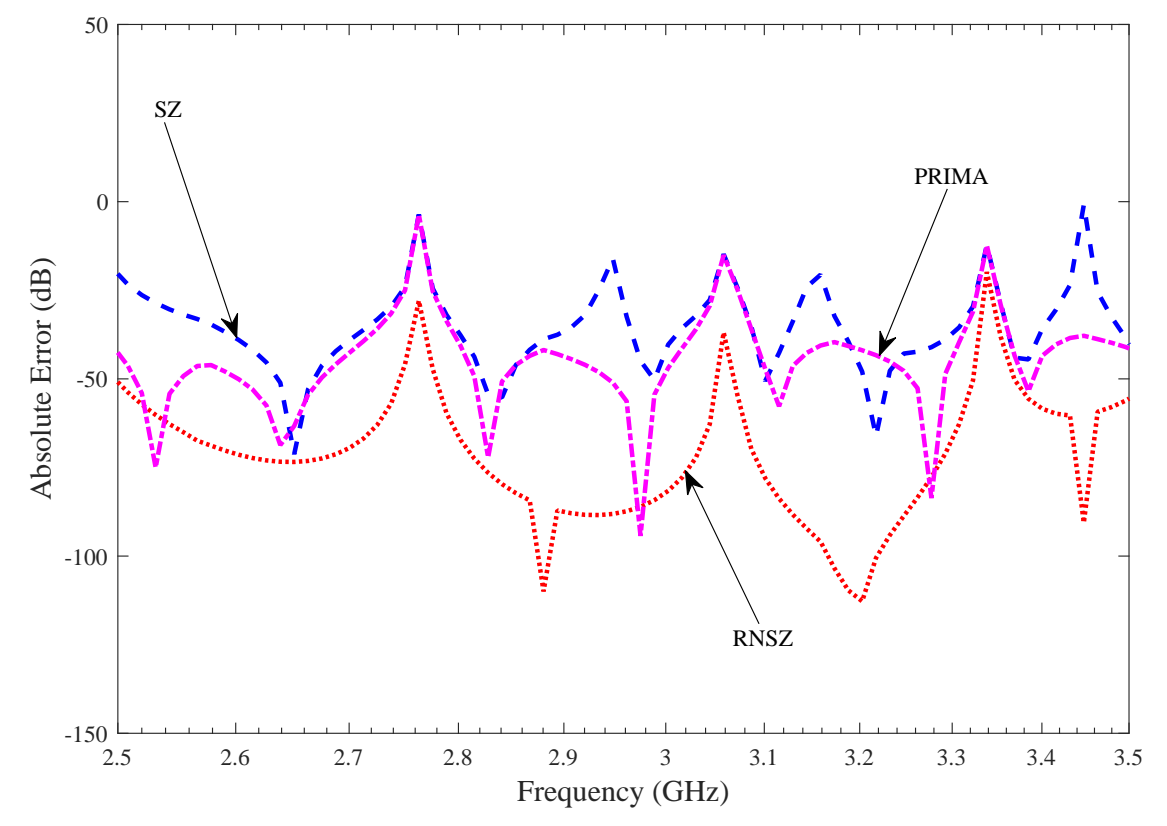

Figure 5. The absolute error plot shows minimum error for the RNSZ method. The calculated mean absolute error for the RLC network in the frequency of interest $(2.5 \mathrm{GHz}$ to $3.5 \mathrm{GHz})$ is $-71.64 \mathrm{~dB}$, $-35.89 \mathrm{~dB}$ and $-44.85 \mathrm{~dB}$ for RNSZ, SZ and PRIMA methods respectively. 


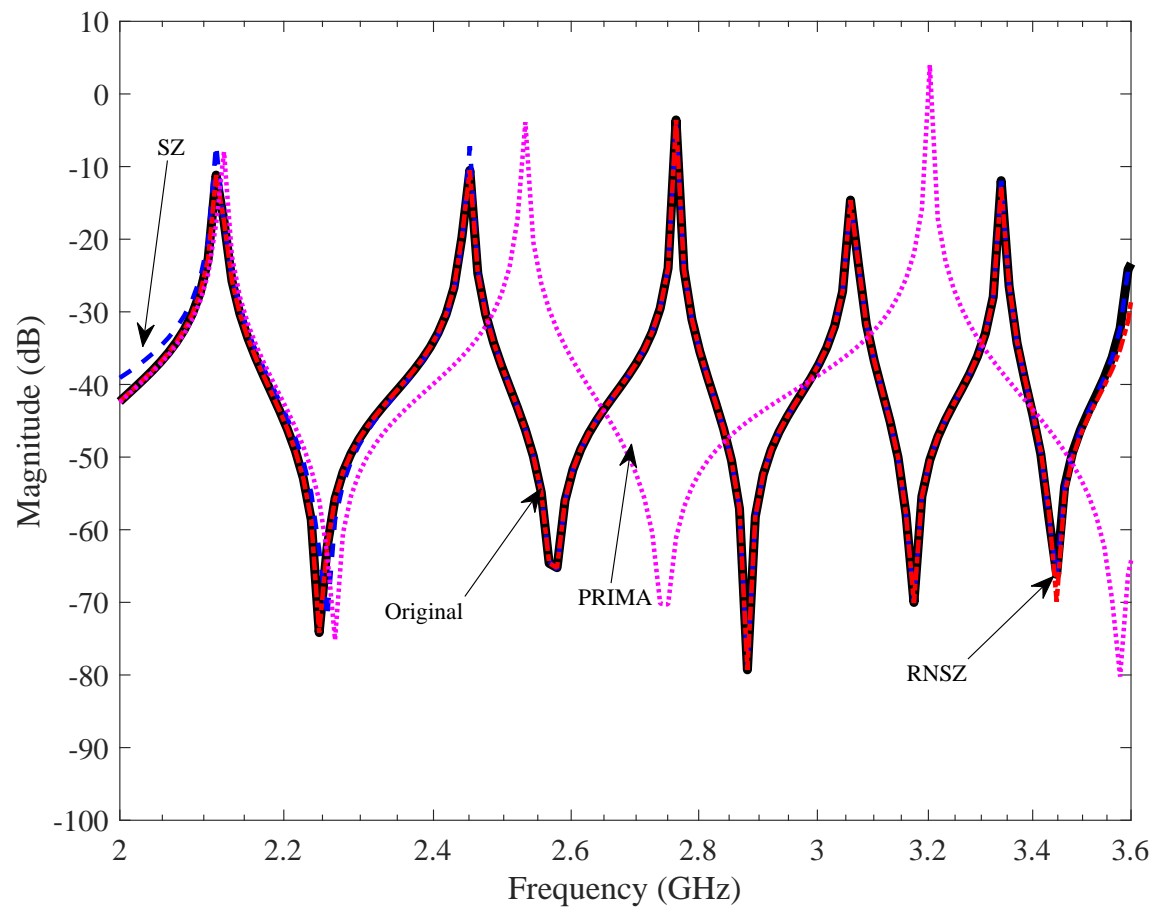

Figure 6. Magnitude response of RLC network for a large span of frequency ( $2 \mathrm{GHz}$ to $3.5 \mathrm{GHz}$ ). The complexity of the reduce order model increased from $\mathrm{N}=10$ to 20 .

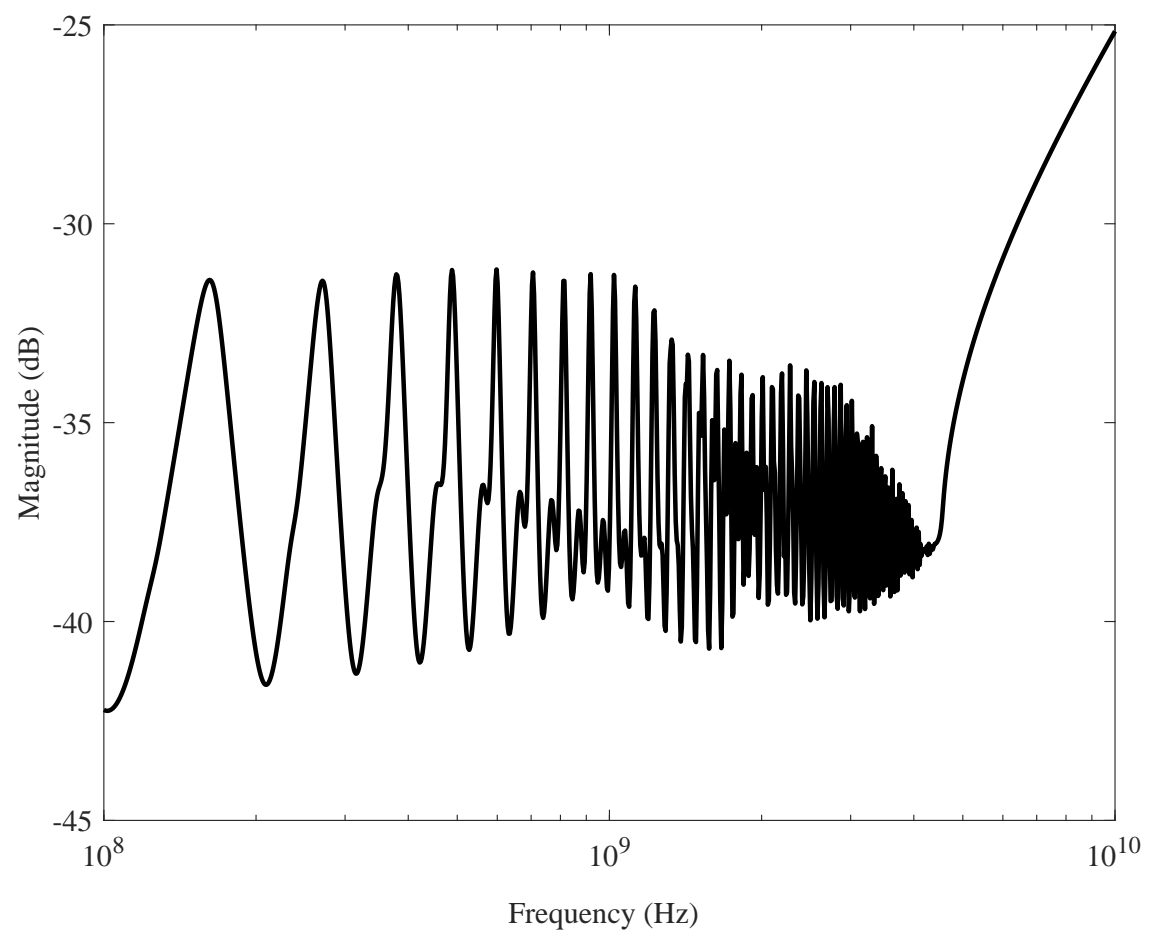

Figure 7. Original system frequency response generated by a interconnect bus of order $n=1076$. 


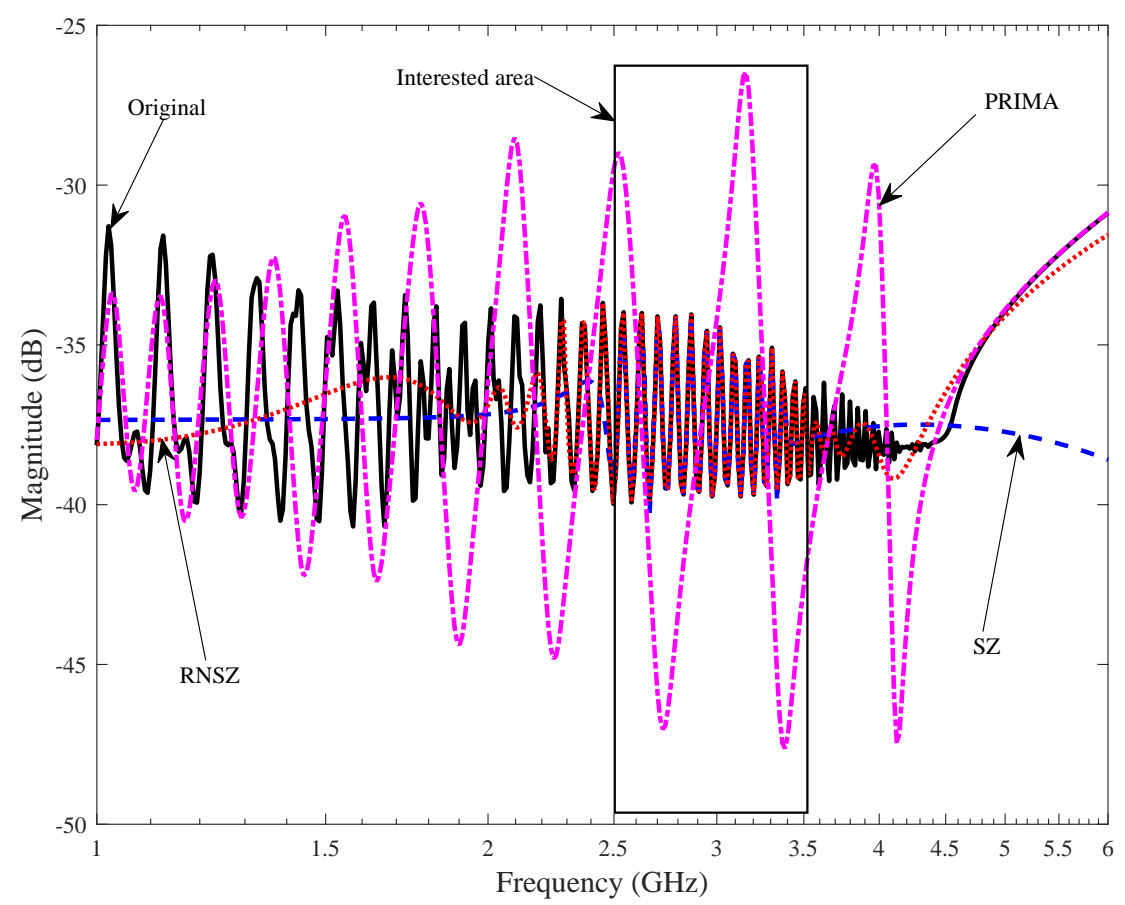

Figure 8. System frequency response of a interconnect bus with original system order $\mathrm{n}=1076$. The reduced order model (ROM) of order $\mathrm{N}=100$ for the other methods shows the response using RNSZ completely matches the original system.

The result of the comparison in Figures 8 and 9 shows that a reduce order 100 using proposed RNSZ method correctly captures the behaviour of the system. However, the PRIMA implementation misses most of the resonance peaks and does not accurately characterize the original system response. It is observed that the Arnoldi based PRIMA matches the low frequency response first and a reduced order 100 is not sufficient to accurately cover the selected frequency range. The error plot shown in Figure 10 highlights the strength of our proposed method (RNSZ) showing the minimum error over the entire range of frequency, i.e., from $2.5 \mathrm{GHz}$ to $3.5 \mathrm{GHz}$. Note that the reduce error is due to the dynamical selection of SZs based on reduce-norm criterion proposed in Section 3. The selection of SZ methods based on interpolation at the selected spectral zeros [37] performs better than PRIMA. However, Figure 10 shows that the calculated mean absolute error for the interconnect bus in the frequency of interest is $-180.52 \mathrm{~dB},-112.93 \mathrm{~dB}$ and $-42.61 \mathrm{~dB}$ for the RNSZ, SZ and PRIMA methods respectively.

In order to show that the proposed RNSZ also works well with large span of frequency, we selected an on-chip spiral inductor example. The original system of order $n=1008$ along with ROM of order $\mathrm{N}=50$ produced by RNSZ and PRIMA methods are plotted in Figure 11. The results clearly show a close match of RNSZ method. However, the PRIMA method show deviations of the magnitude plot compared to original system and missed out most of the peaks of the system transfer function. The simulation example clearly show validity of the proposed RNSZ method for large span of frequency. Note that the order and hence the number of states required to represent the ROM will increase with large frequency of interest and a more complex example. 


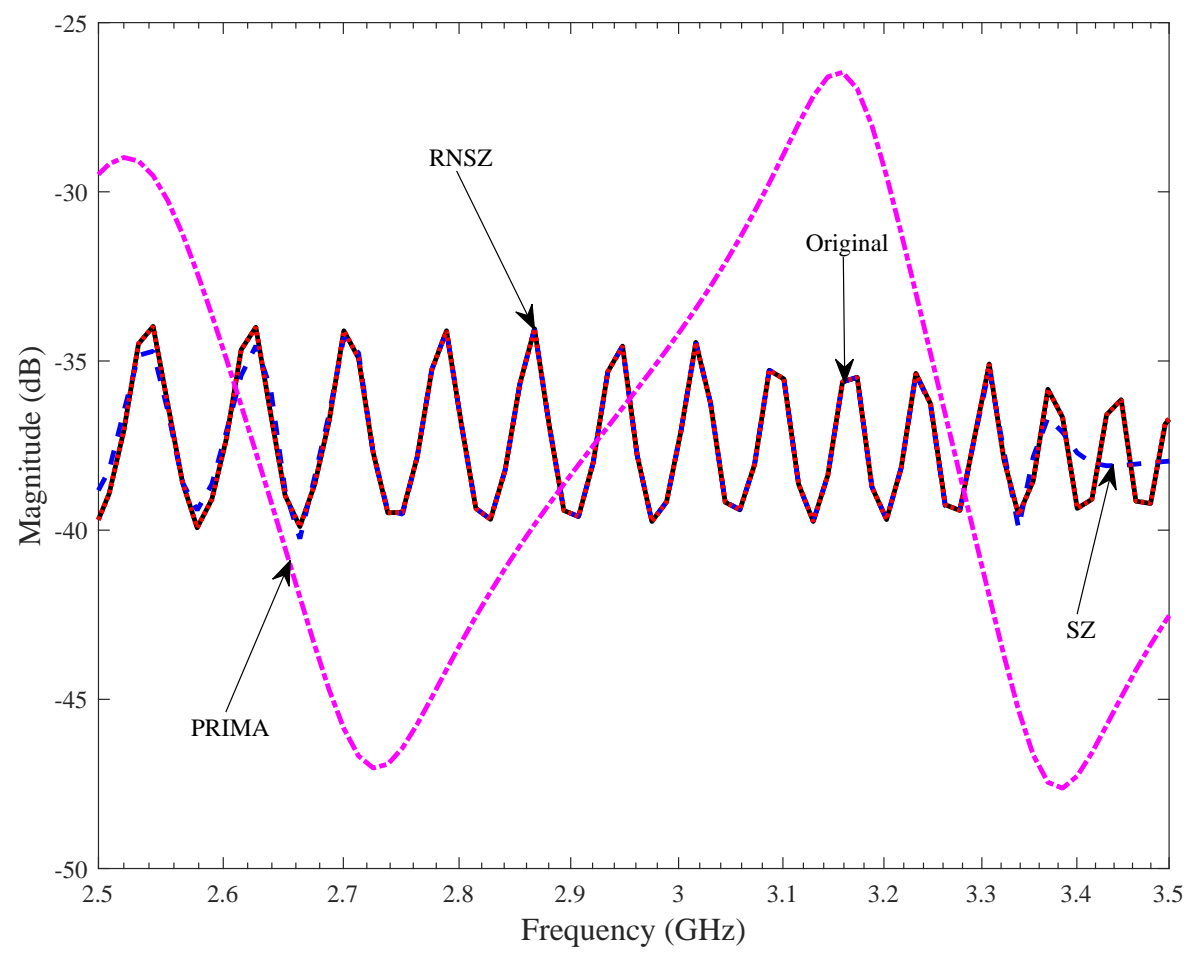

Figure 9. Magnitude response for the frequency of interest (2.5 GHz to $3.5 \mathrm{GHz}$ ) for an Intel Core i7-2920XM processor interconnect bus. The proposed method (RNSZ) closely matches the fine details of the original on-chip interconnect bus.

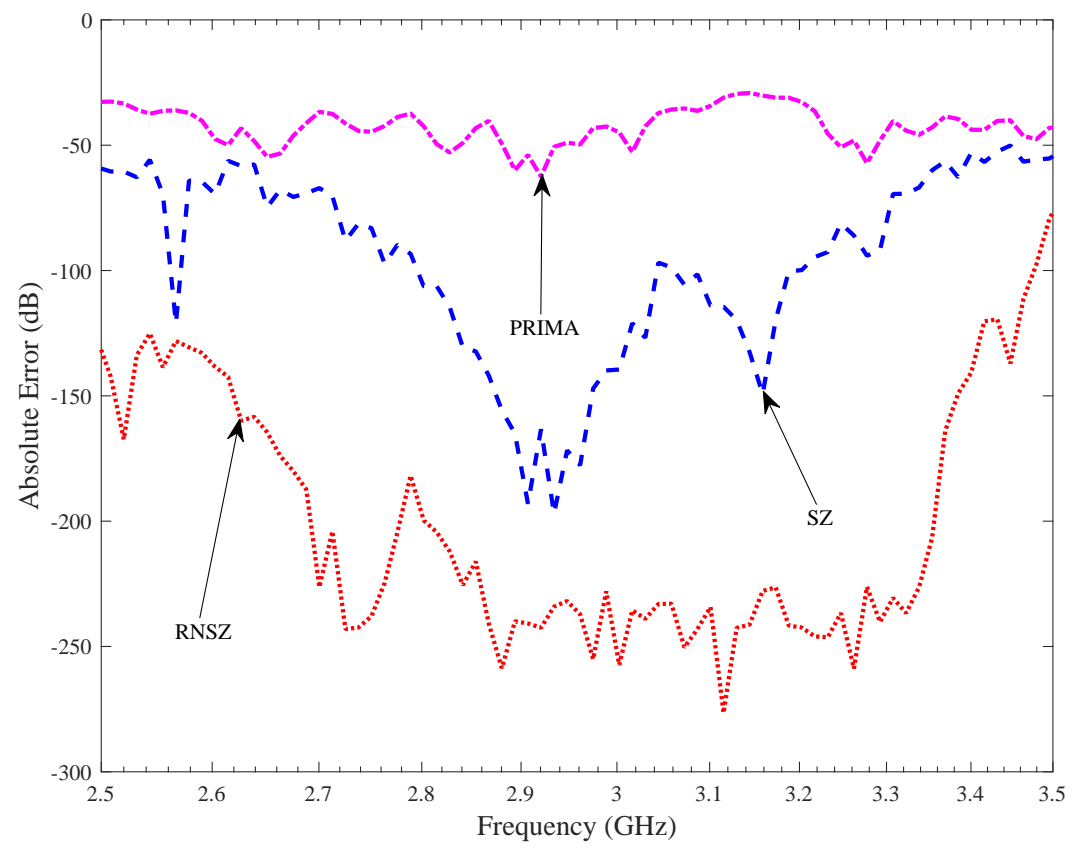

Figure 10. The absolute error plot shows minimum error for the RNSZ method. The calculated mean absolute error for the interconnect bus example in the frequency of interest is $-180.52 \mathrm{~dB},-112.93 \mathrm{~dB}$ and $-42.61 \mathrm{~dB}$ for RNSZ, interpolation by SZ and PRIMA methods respectively. 


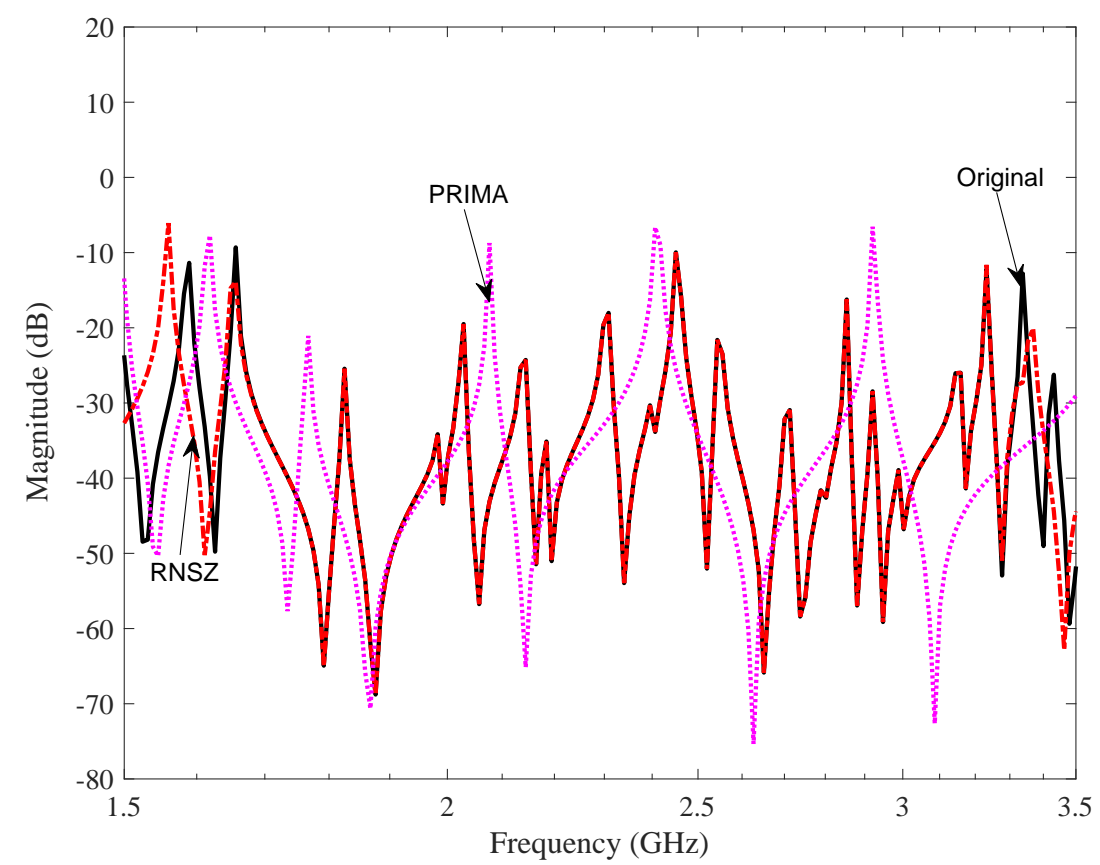

Figure 11. System frequency response of a spiral inductor with original system order of $n=1008$. The selected large span of frequency now cover over $2 \mathrm{GHz}$ of frequency range, i.e., from $1.5 \mathrm{GHz}$ to $3.5 \mathrm{GHz}$. A minimum ROM of order $\mathrm{N}=50$ is required by RNSZ method to match the response. However, with similar ROM $(\mathrm{N}=50)$, the PRIMA method missed out most of the response and peaks of the original system.

A comparison of the mean absolute error of the ROM generated by the proposed RNSZ, PRIMA and SZ methods are tabulated in Table 1. The calculated mean absolute errors for the RLC network and Interconnect bus shows that the proposed RNSZ correctly captures the behaviour of the system with almost negligible error.

Table 1. Mean absolute error comparison for various examples.

\begin{tabular}{lccc}
\hline Examples & PRIMA [19] & SZ [37] & Proposed RNSZ \\
\hline RLC network & $-44.85 \mathrm{~dB}$ & $-35.89 \mathrm{~dB}$ & $-71.64 \mathrm{~dB}$ \\
Interconnect bus & $-42.61 \mathrm{~dB}$ & $-112.93 \mathrm{~dB}$ & $-180.52 \mathrm{~dB}$ \\
Spiral inductor & $-37.97 \mathrm{~dB}$ & - & $-177.28 \mathrm{~dB}$ \\
\hline
\end{tabular}

In the end, we take series of examples generated from an on-chip spiral inductor with transfer function spanning from few $\mathrm{kHz}$ to $\mathrm{GHz}$ range as shown in Figure 12. In first example, we take the frequency range from $100 \mathrm{MHz}$ to $3 \mathrm{GHz}$ and plot the original and ROM function (order $\mathrm{n}=38$ ) in Figure 13. In this simulation, we selected lower and upper range of frequencies to be $100 \mathrm{MHz}$ and $3 \mathrm{GHz}$ respectively. The absolute error plot in Figure 14 shows a close match with a mean absolute error of $-129.98 \mathrm{~dB}$ in the selected range of frequencies. The second example is the same spiral inductor, however this time, we selected the lower and upper range of frequencies to be $10 \mathrm{MHz}$ and $3 \mathrm{GHz}$ respectively. The simulation results are plotted in Figure 15, which shows a close match with ROM of order $n=50$ computed using RNSZ method. The absolute error is shown in Figure 16 with a mean absolute error of $-100.97 \mathrm{~dB}$ in the selected range of frequencies. It is important to note that these two examples cover narrow band of frequencies and their simulation results shows overlapping peaks with their mean absolute error less than $-100 \mathrm{~dB}$.

In order to gain good inside of the problem related with large band of frequencies, the next two examples are spread over a large span of frequencies, i.e., $1 \mathrm{MHz}$ to $3 \mathrm{GHz}$ and $3 \mathrm{kHz}$ to $3 \mathrm{GHz}$. In third 
example we selected lower and upper range of frequencies to be $1 \mathrm{MHz}$ and $3 \mathrm{GHz}$ respectively. The simulation results are plotted in Figure 17 with a reduce order of $n=180$ matching the original frequency response. It is worth mentioning here that ROM order in this case is quite high, almost comparable to the order of the original system. Therefore, the example highlights that the proposed RNSZ techniques is not suitable for large span of frequencies. The absolute error plot in Figure 18 shows a good match with a mean absolute error of $-73.98 \mathrm{~dB}$ in the selected range of frequencies. The fourth and last example is for a frequency range of $3 \mathrm{kHz}$ to $3 \mathrm{GHz}$. The simulation results are plotted in Figure 19 and again a very high reduce order of $n=186$ is required to match the original frequency response. The absolute error plot in Figure 18 shows a good match with a mean absolute error of $-76.29 \mathrm{~dB}$ in the selected range of frequencies.

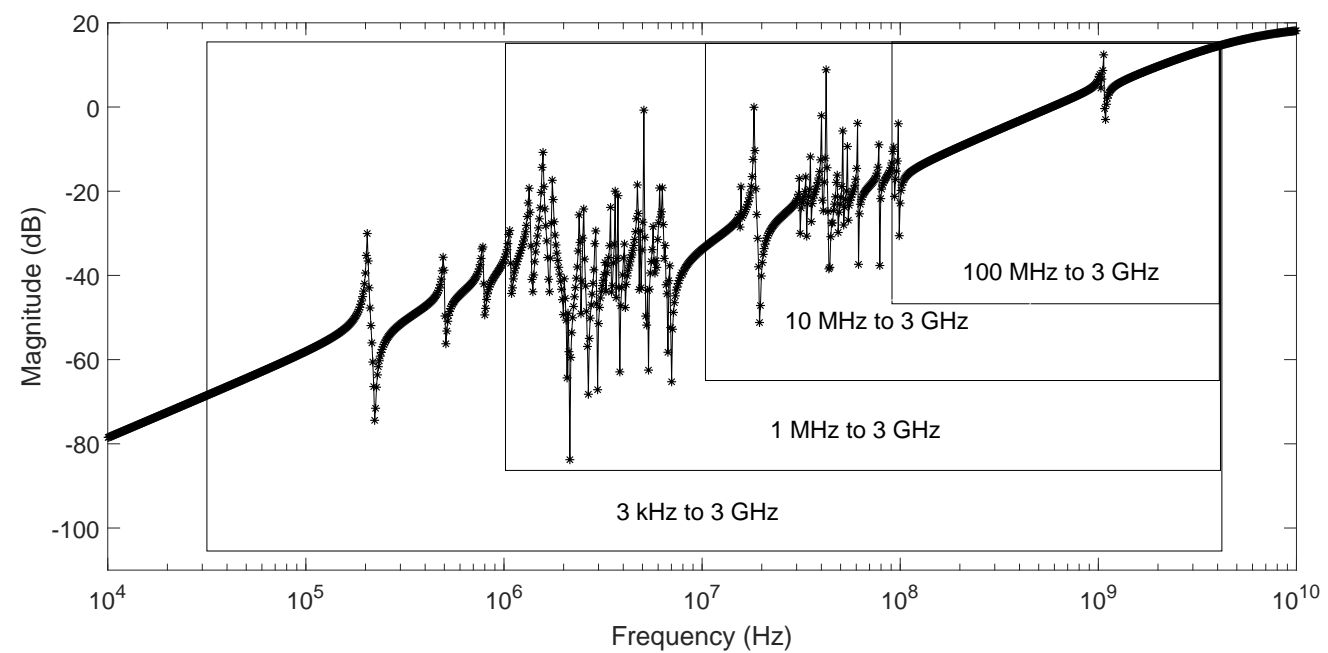

Figure 12. System frequency response of a spiral inductor with original system order of $n=191$. The selected spans of frequency now cover different frequency ranges from $3 \mathrm{kHz}$ to $3 \mathrm{GHz}$.

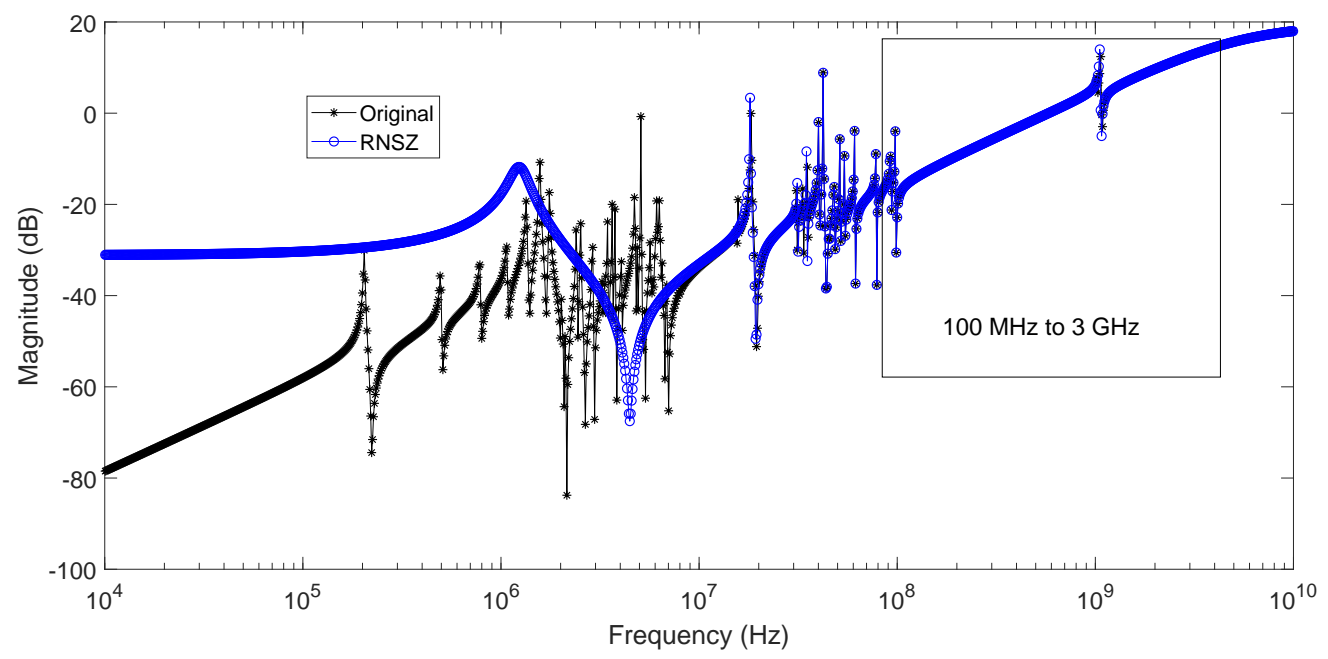

Figure 13. Original system frequency response of a spiral inductor (order $n=191$ ) compared with a ROM generated using RNSZ of order $\mathrm{N}=38$ covering $100 \mathrm{MHz}$ to $3 \mathrm{GHz}$ frequencies. 


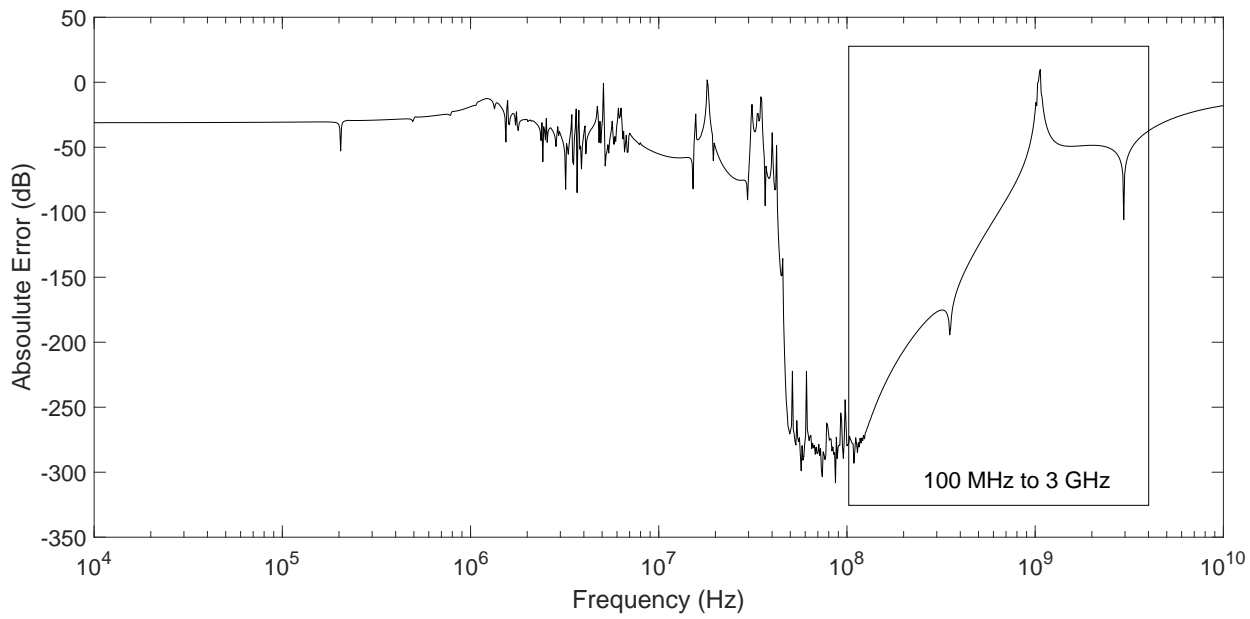

Figure 14. Absolute error of ROM generated using RNSZ of order $\mathrm{N}=38$ compared to original system frequency response of a spiral inductor (order $n=191$ ). The mean absolute error is $-129.98 \mathrm{~dB}$ from $100 \mathrm{MHz}$ to $3 \mathrm{GHz}$ frequencies.

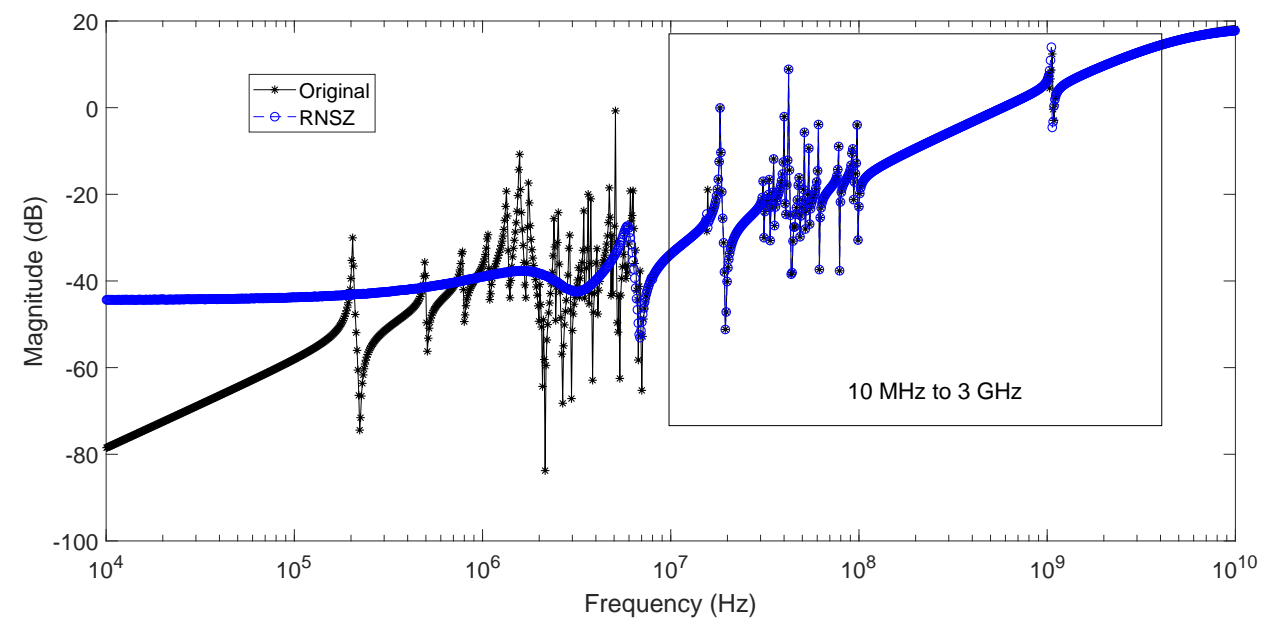

Figure 15. Original system frequency response of a spiral inductor (order $n=191$ ) compared with a ROM generated using RNSZ of order $\mathrm{N}=50$ covering $10 \mathrm{MHz}$ to $3 \mathrm{GHz}$ frequencies.

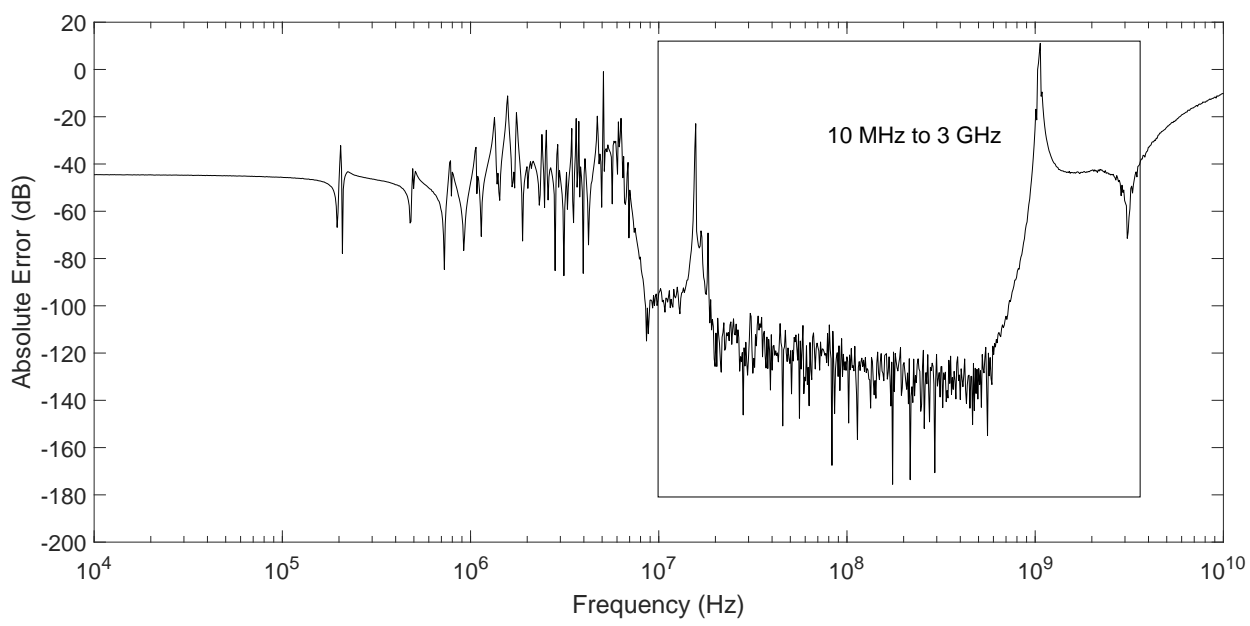

Figure 16. Absolute error of ROM generated using RNSZ of order $\mathrm{N}=50$ compared to original system frequency response of a spiral inductor (order $n=191$ ). The mean absolute error is $-100.97 \mathrm{~dB}$ from $10 \mathrm{MHz}$ to $3 \mathrm{GHz}$ frequencies. 


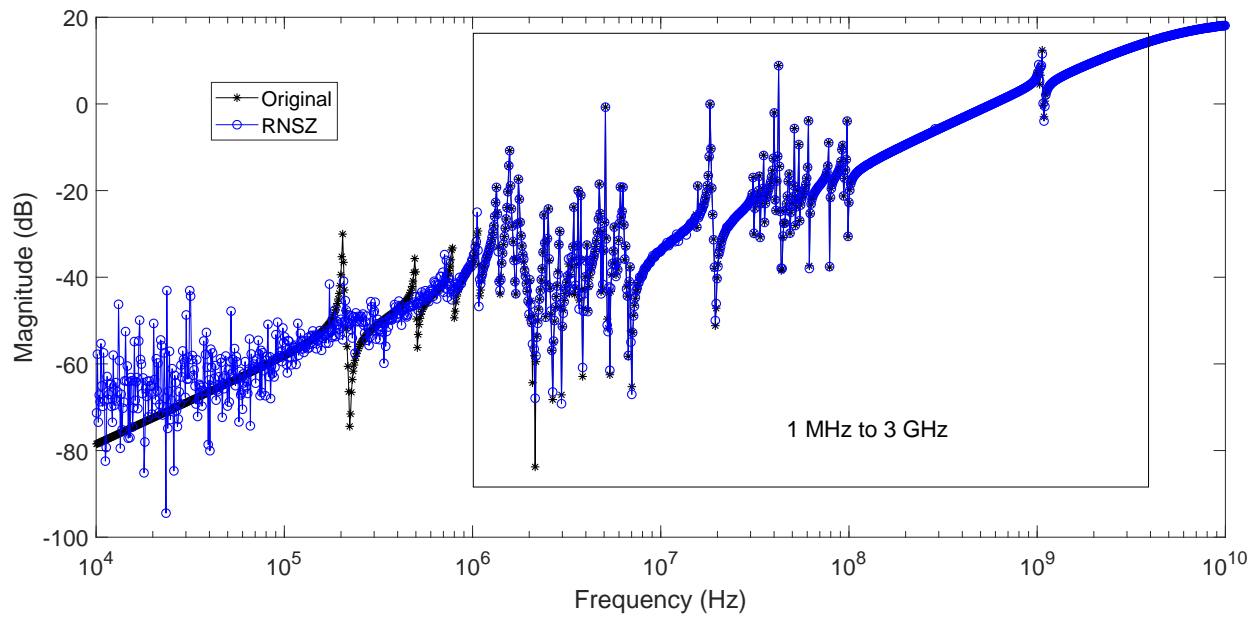

Figure 17. Original system frequency response of a spiral inductor (order $n=191$ ) compared with a ROM generated using a high order $(\mathrm{N}=180)$ RNSZ method covering $1 \mathrm{MHz}$ to $3 \mathrm{GHz}$ frequencies.

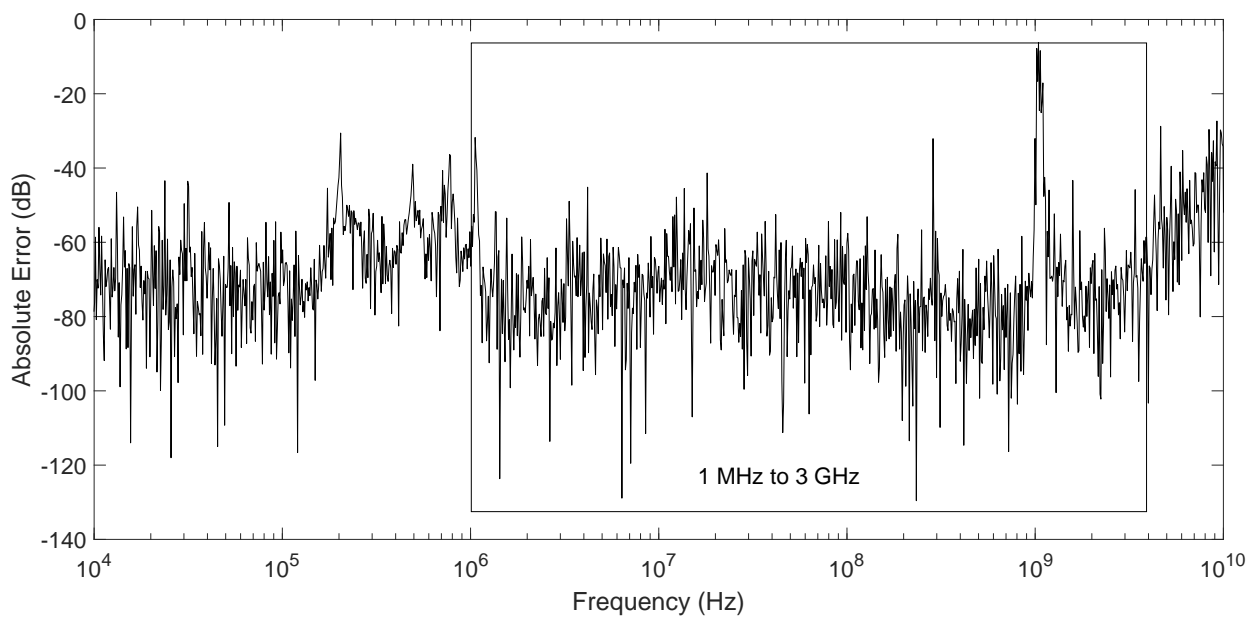

Figure 18. Absolute error of ROM generated using RNSZ of order $N=180$ compared to original system frequency response of a spiral inductor (order $n=191$ ). The mean absolute error is $-73.98 \mathrm{~dB}$ from 1 $\mathrm{MHz}$ to $3 \mathrm{GHz}$ frequencies.

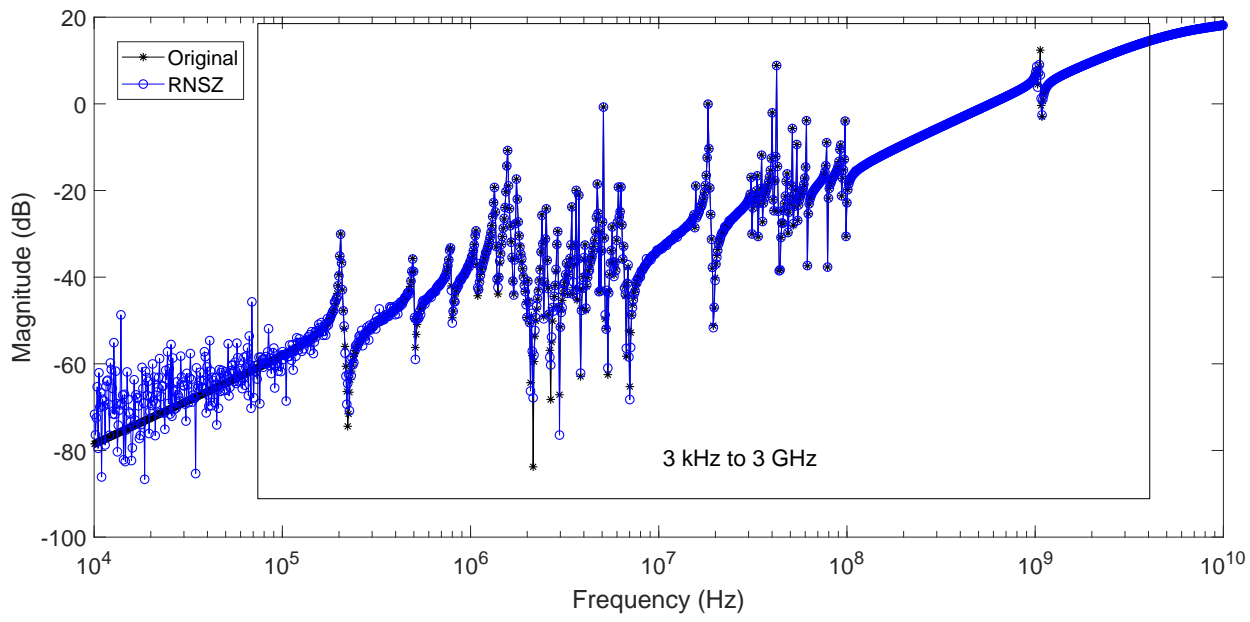

Figure 19. Original system frequency response of a spiral inductor (order $n=191$ ) compared with a ROM generated using a high order $(\mathrm{N}=186) \mathrm{RNSZ}$ method covering $3 \mathrm{kHz}$ to $3 \mathrm{GHz}$ frequencies. 
In the end, Table 2 summarizes the results to compare accuracy of RNSZ simulation over large band of frequencies. The on-chip spiral inductor example, with transfer function plotted in Figure 12, spans frequencies from few $\mathrm{kHz}$ to $\mathrm{GHz}$. Its simulation results are plotted from Figures 13-20 and are included in Table 2. The table compares ROM accuracy and complexity for different range of frequencies.

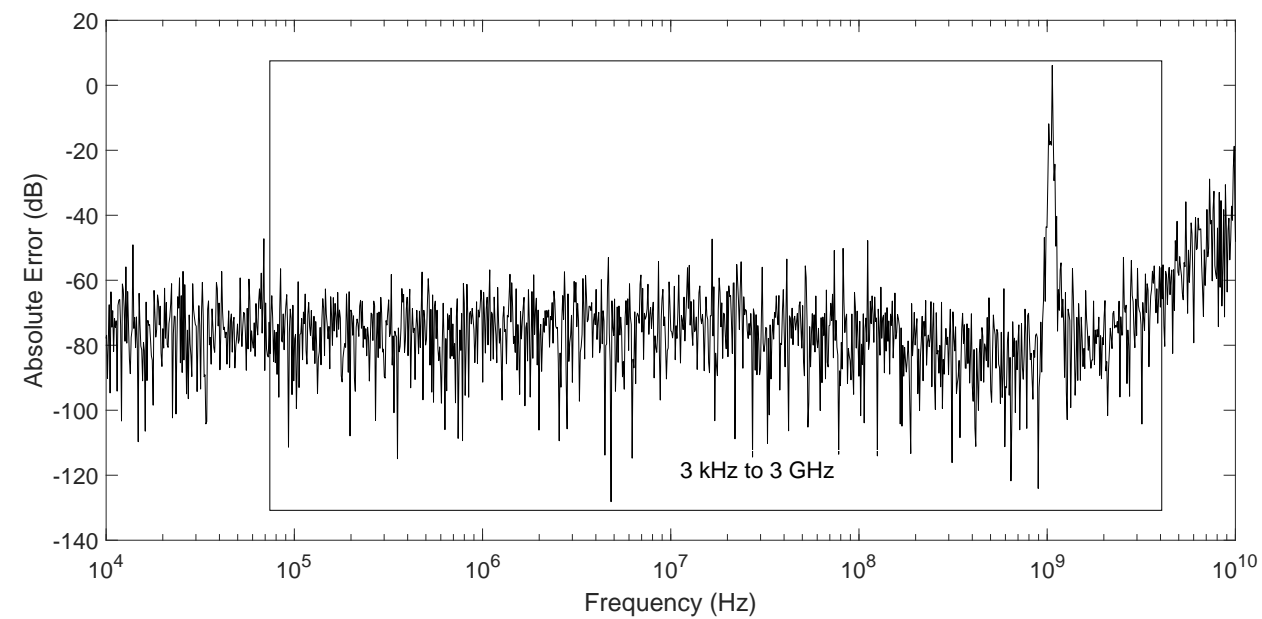

Figure 20. Absolute error of ROM generated using RNSZ of order $\mathrm{N}=186$ compared to original system frequency response of a spiral inductor (order $n=191$ ). The mean absolute error is $-76.29 \mathrm{~dB}$ from $3 \mathrm{kHz}$ to $3 \mathrm{GHz}$ frequencies.

Table 2. Comparison of spiral inductor example (Figure 12 with original system order $n=191$ ).

\begin{tabular}{lccl}
\hline Frequency Range & Reduce Order & Mean Absolute Error & Remarks \\
\hline $3 \mathrm{kHz}-3 \mathrm{GHz}$ & 186 & $-76.29 \mathrm{~dB}$ & Matches original function with high order ROM \\
$1 \mathrm{MHz}-3 \mathrm{GHz}$ & 180 & $-73.98 \mathrm{~dB}$ & Matches original function with high order ROM \\
$10 \mathrm{MHz}-3 \mathrm{GHz}$ & 50 & $-100.97 \mathrm{~dB}$ & Closely matches original function \\
$100 \mathrm{MHz}-3 \mathrm{GHz}$ & 38 & $-129.98 \mathrm{~dB}$ & Closely matches original function \\
\hline
\end{tabular}

It is important to note that for large span of frequencies (row 1 and 2 of Table 2), the order of the ROMs are quite high, which signifies no real advantage gain by model order reduction of the original system. However, for small span of frequencies (row 3 and 4 of Table 2), the results of RNSZ method gives greater accuracy with fairly small ROMs in addition to guaranteeing stability and passivity of systems. The results discussed in this section and Table 2 clearly highlight the fact that RNSZ is very efficient for application specific solutions, i.e., for narrow band of frequency with span ranging in few $\mathrm{GHz}$ and its use may be restricted only for high-speed applications.

\section{Conclusions}

Over the last few decades, scaling in deep submicron had shifted the paradime from device-dominated to interconnect-dominated design methodology. In this work, we developed a new reduce-norm frequency selective projection method by using interpolation point based on SZs of the system. The proposed reduce-norm scheme dynamically selects spectral zeros, which can guarantee stability and passivity, while creating the ROMs accurate across selected narrow range of frequencies for application specific MOR. 
Author Contributions: Conceptualization, M.A.; Formal analysis, N.A., M.A. and R.M.; Funding acquisition, R.H., A.A., S.M., N.A., R.M. and A.U.H.; Investigation, N.A. and M.A.; Methodology, N.A. and M.A.; Project administration, A.U.H.; Software, N.A., and M.A.; Supervision, M.A.; Validation, N.A., M.A. and R.M.; Writing—original draft, M.A. and N.A.; Writing—review \& editing, M.A., N.A., R.H., A.A. and S.M. All authors have read and agreed to the published version of the manuscript.

Funding: The authors extend their appreciation to the Technical University of Munich (TUM), Mirpur University of Science and Technology (MUST), Mirpur 10250, Pakistan and Deanship of Scientific Research at King Saud University for funding this work through research group no.RG-1441-351.

Conflicts of Interest: The authors declare no conflict of interest.

\section{Abbreviations}

The following abbreviations are used in this manuscript:

$\mathbb{R} \quad$ The set of real numbers

$\mathbb{C} \quad$ The set of complex numbers, i.e., s-plane

$\Lambda \quad$ Eigenvalues

SZ Spectral Zeros

MOR Model Order System

ROM Reduced Order Model

RNSZ Reduced Norm Spectral Zero

LTIs Linear Time Invariant system

\section{References}

1. Ciuprina, G.; Ioan, D. Scientifc Computing in Electrical Engineering; SCEE ser. Mathematics in Industry; Springer: Berlin, Germany, 2007.

2. Wan, F.Y. Mathematical Models and Their Analysis; SIAM: Philadelphia, PA, USA, 2018; p. 79.

3. Kundu, S.; Chattopadhyay, S. Network-On-Chip: The Next Generation of System-on-Chip Integration; CRC Press: Boca Raton, FL, USA, 2018.

4. Gugulothu, B.; Naik, B.R.; Boodidha, S. Modeling of Capacitive Coupled Interconnects for Crosstalk Analysis in High Speed VLSI Circuits. In Proceedings of the 2019 International Conference on Communication and Signal Processing (ICCSP), Chennai, India, 4-6 April 2019.

5. Mitra, P.; Sharma, B.; Chandna, V.K.; Rathore, V.S. Design and Performance Evaluation of Hybrid Wired-Wireless Network on Chip Interconnect Architectures. In Third International Congress on Information and Communication Technology; Springer: Singapore, 2019; pp. 191-199.

6. Ayala, J.L. (Ed.) Communication Architectures for Systems-On-Chip; CRC Press: Boca Raton, FL, USA, 2018.

7. Alam, M.; Nieuwoudt, A.; Massoud, Y. Wavelet-Based Interpolation Point Selection for Multi-Shifted Arnoldi. In Proceedings of the 2007 IEEE International Symposium on Circuits and Systems, New Orleans, LA, USA, 27-30 May 2007; pp. 653-656.

8. Silveira, L.M.; Kamon, M.; White, J. Efficient reduced-order modeling of frequency-dependent coupling inductances associated with 3-D interconnect structures. IEEE Trans. Components Packag. Manuf. Technol. Part $B$ 1996, 19, 283-288. [CrossRef]

9. Alam, M.; Nieuwoudt, A.; Massoud, Y. Wavelet-based passivity preserving model order reduction for wideband interconnect characterization. In Proceedings of the 8th International Symposium on Quality Electronic Design (ISQED’07), San Jose, CA, USA, 26-28 March 2007; pp. 432-437.

10. Wang, X.; Yu, M.; Wang, C. Structure-Preserving-Based Model-Order Reduction of Parameterized Interconnect Systems. Circuits, Syst. Signal Process. 2018, 37, 19-48. [CrossRef]

11. Gugercin, S. Projection Methods for Model Reduction of Large-Scale Dynamical Systems. Ph.D. Thesis, Rice University, Houston, TX, USA, 2003.

12. Wang, X.L.; Jiang, Y.L. Two-sided projection methods for model reduction of MIMO bilinear systems. Math. Comput. Model. Dyn. Syst. 2013, 19, 575-592. [CrossRef]

13. Alam, M. Compact Models for nanophotonic Structures and On-Chip Interconnects. Ph.D. Thesis, Rice University, Houston, TX, USA, 2007. 
14. Chaudhary, S.K.; Kumar, A. Hankel norm approximation of a stable non-minimal system. In Proceedings of the 1st International Conference on Electronics, Materials Engineering and Nano-Technology, Science City, Kolkata, India, 28-29 April 2017; pp. 1-4.

15. Antoulas, A.C. Approximation of Large-Scale Dynamical Systems; SIAM: Philadelphia, PA, USA, 2005.

16. Alam M.; Nieuwoudt A.; Massoud Y. Reduced-Order Wide-Band Interconnect Model Realization using Filter-Based Spline Interpolation. In Proceedings of the 2007 Asia and South Pacific Design Automation Conference, Yokohama, Japan, 23-26 January 2007; pp. 373-378.

17. Zulfiqar, U.; Tariq, W.; Li, L.; Liaquat M. A Passivity-Preserving Frequency-Weighted Model Order Reduction Technique. IEEE Trans. Circuits Syst. II 2017, 64, 1327-1331. [CrossRef]

18. Sheldon, L.H.; Tan, X.D. Advanced Model Order Reduction Techniques in VLSI Design; Cambridge University Press: Cambridge, UK, 2007.

19. Odabasioglu, A.; Celik, M.; Pileggi, L.T. PRIMA: Passive Reduced-Order InterconnectMacromodeling 194 Algorithm. IEEE Trans. CAD 1998, 17, 645-654. [CrossRef]

20. Alam, M.; Nieuwoudt, A.; Massoud, Y. Model order reduction using spline-based dynamic multi-point rational interpolation for passive circuits. Analog. Integr. Circuits Signal Process. 2007, 50, 273-277. [CrossRef]

21. Freund, R.W. Krylov-subspace methods for reduced-order modeling in circuit simulation. J. Comput. Appl. Math. 2000, 123, 395-421. [CrossRef]

22. Freund, R. Sprim: Structure-preserving reduced-order interconnect macromodeling. In Proceedings of the IEEE/ACM International Conference on Computer Aided Design, San Jose, CA, USA, 7-11 November 2004; pp. 80-87.

23. Alam, M.; Nieuwoudt, A.; Massoud, Y. Efficient multi-shifted Arnoldi projection using wavelet transform. J. Circuits, Syst. Comput. 2007, 16, 699-709. [CrossRef]

24. Alam, M.; Nieuwoudt, A.; Massoud, Y. Dynamic Multi-Point Rational Interpolation for Frequency-Selective Model Order Reduction. In Proceedings of the 2006 IEEE Dallas/CAS Workshop on Design, Applications, Integration and Software, Richardson, TX, USA, 29-30 October 2006; pp. 95-98.

25. Alam, M.; Nieuwoudt, A.; Massoud, Y. Provably passive second order model order reduction for package parasitics using spectral zeros. In Proceedings of the 2007 IEEE Northeast Workshop on Circuits and Systems, Montreal, QC, Canada, 5-8 August 2007; pp. 791-794.

26. Silveira, L.M.; Kamon, M.; Elfadel, I.; White, J. A Coordinate Transformed Arnoldi Algorithm for Generating Guaranteed Stable Reduced Order Models of RLC Circuits. In Proceedings of the International Conference on Computer Aided Design, San Jose, CA, USA, 10-14 November 1996; pp. 288-294.

27. Zheng, H.; Pileggi, L.T. Robust and Passive Model Order Reduction for Circuits Containing Susceptance Elements. In Proceedings of the IEEE/ACM International Conference on Computer Aided Design, San Jose, CA, USA, 10-14 November 2002; pp. 761-766.

28. Schilders, W.H.; Van der Vorst, H.A.; Rommes, J. Model Order Reduction, Theory, Research Aspects and Applications; Springer: Berlin, Germany, 2008.

29. Phillips, J.; Silveira, L.M. Poor Man's TBR: A Simple Model Reduction Scheme. IEEE Trans. Comput. Aided Des. Integr. Circuits Syst. 2005, 24, 43-55. [CrossRef]

30. Silveira, L.M.; Phillips, J. Exploiting Input Information in a Model Reduction Algorithm for Massively Coupled Parasitic Networks. In Proceedings of the 41st Design Automation Conference, San Diego, CA, USA, 7-11 July 2004; pp. 385-388.

31. Phillips, J.; Daniel, L.; Silveira, M. Guaranteed Passive Balancing Transformations for Model Order Reduction. In Proceedings of the 2002 Design Automation Conference, New Orleans, LA, USA, 10-14 June 2002; pp. 52-57.

32. Su, Q.; Balakrishnan, V.; Koh, C.K. Efficient Approximate Balanced Truncation of General Large-Scale RLC Systems via Krylov Methods. In Proceedings of the 15th International Conference of Asia and South Pacific Design Automation on VLSI Design, Bangalore, India, 7-11 January 2002; pp. 311-316.

33. Ismail, Y. Evaluating Noise Pulses in RC Networks due to Capacitive Coupling. In Proceedings of the 2002 IEEE International Symposium on Circuits and Systems, Phoenix-Scottsdale, AZ, USA, 26-29 May 2002; pp. 653-656.

34. Vogel, C.R.; Wade, J.G. Iterative SVD-based methods for ill-posed problems. SIAM J. Sci. Comput. 1994, 15, 736-754. [CrossRef] 
35. Antoulas, A.C. A new result on passivity preserving model reduction. Syst. Control. Lett. 2005, 54, 361-374. [CrossRef]

36. Alam M.; Nieuwoudt A.; Massoud Y. Frequency selective model order reduction via spectral zero projection. In Proceedings of the 2007 Asia and South Pacific Design Automation Conference, Yokohama, Japan, 23-26 January 2007; pp. 379-383.

37. Sorensen, D. Passivity preserving model reduction via interpolation of spectral zeros. Syst. Control Lett. 2005, 54, 347-360. [CrossRef]

38. Ionutiu, R.; Rommes, J.; Antoulas, A.C. Passivity-preserving model reduction using dominant spectral-zero interpolation. IEEE Trans. Comput. Aided Des. Integr. Circuits Syst. 2008, 27, 2250-2263. [CrossRef]

39. Mondal M.; Massoud, Y. Reducing Pessimism in RLC Delay Estimation Using an Accurate Analytical Frequency Dependent Model for Inductance. In Proceedings of the IEEE/ACM International Conference on Computer-Aided Design, San Jose, CA, USA, 6-10 November 2005; pp. 690-695.

40. Wang, J.M.; Chu, C.-C.; Yu,Q.; Kuh, E.S. On Projection-Based Algorithms for Model-Order Reduction of Interconnects. IEEE Trans. Circuits Syst.- I 2002, 49, 1563-1585. [CrossRef]

41. Kamon, M.; Wang, F.; White, J. Generating Nearly Optimally Compact Models from Krylov-Subspace Based Reduced-Order Models. IEEE Trans. Circuits Syst.- II 2000, 47, 239-248. [CrossRef]

42. Benini, L.; DeMicheli, G. Dynamic Power Management: Design Techniques and CAD Tools; Springer Science and Business Media: Berlin, Germany, 2012.

43. Ionutiu, R.; Lefteriu, S.; Antoulas A. Comparison of model reduction methods with applications to circuit simulation. In Scientific Computing in Electrical Engineering; Springer: Berlin, Germany, 2007; Volume 11.

44. Ionutiu, R. Model Reduction for Descriptor Systems with Applications to Circuit Simulation; William Marsh Rice University: Houston, TX, USA, 2007; Rep. TREE07-07.

45. Mehrmann, V.; Stykel, T.; Benner, P.; Sorensen, D. Balanced Truncation Model Reduction for Large-Scale Systems in Descriptor Form, in Dimension Reduction of Large-Scale Systems; Ser. LNCSE; Springer: Berlin, Germany, 2005; p. 45.

46. Charles, J.; Jassi, P.; Ananth, N.S.; Sadat, A.; Fedorova, A. Evaluation of the Intel Core $^{\mathrm{TM}}$ i7 turbo boost feature. In Proceedings of the IEEE International Symposium on Workload Characterization, Austin, TX, USA, 4-6 October 2009; pp. 188-197.

47. Nabors, K.; White, J. FastCap: A multipole accelerated 3-D capacitance extraction program. IEEE Trans. Comput.-Aided Des. 1991, 10, 1447-1459. [CrossRef]

48. Kamon, M.; Tsuk, M.J.; White, K. FASTHENRY: A multipole-accelerated 3-D inductance extraction program. IEEE Trans. Microwave Theory Tech. 1994, 42, 1750-1758. [CrossRef] 